\title{
STABILITY OF ANOSOV HAMILTONIAN STRUCTURES
}

\author{
WILL J. MERRY AND GABRIEL P. PATERNAIN
}

\begin{abstract}
Let $\left(M^{n}, g\right)$ denote a closed Riemannian manifold $(n \geq 3)$ which admits a metric of negative curvature (not necessarily equal to $g$ ). Let $\omega_{1}:=$ $\omega_{0}+\pi^{*} \sigma$ denote a twisted symplectic form on $T M$, where $\sigma \in \Omega^{2}(M)$ is a closed 2 -form and $\omega_{0}$ is the symplectic structure on $T M$ obtained by pulling back the canonical symplectic form $d x \wedge d p$ on $T^{*} M$ via the Riemannian metric. Let $\Sigma_{k}$ be the hypersurface $|v|=\sqrt{2 k}$. We prove that if $n$ is odd and the Hamiltonian structure $\left(\Sigma_{k}, \omega_{1}\right)$ is Anosov with $C^{1}$ weak bundles then $\left(\Sigma_{k}, \omega_{1}\right)$ is stable if and only if it is contact. If $n$ is even and in addition the Hamiltonian structure is $1 / 2$-pinched, then the same conclusion holds. As a corollary we deduce that if $g$ is negatively curved, strictly $1 / 4$-pinched and $\sigma$ is not exact then the Hamiltonian structure $\left(\Sigma_{k}, \omega_{1}\right)$ is never stable for all sufficiently large $k$.
\end{abstract}

\section{INTRODUCTION}

Let $\Sigma$ be a closed oriented manifold of dimension $2 n-1$. A Hamiltonian structure on $\Sigma$ is a closed 2-form $\omega$ such that $\omega^{n-1} \neq 0$. Its kernel ker $\omega$ defines an orientable 1-dimensional foliation.

A natural condition to impose on a Hamiltonian structure is stability; this asserts the existence of a 1-form $\lambda$ such that ker $\omega \subseteq$ ker $d \lambda$ and such that $\lambda \wedge \omega^{n-1}>0$. The 1-form $\lambda$ is known as a stabilizing 1-form.

A stronger condition one might like to impose is the following: $(\Sigma, \omega)$ is called of contact type if we can find a 1-form $\lambda$ such that $d \lambda=\omega$ and $\lambda \wedge \omega^{n-1}>0$. In particular $\lambda$ is a stabilizing 1-form and $\lambda$ is a contact form on $\Sigma$. Note that $(\Sigma, \omega)$ can be of contact type only if $\omega$ is exact. The stability condition first appeared in 22] as a condition for which the Weinstein conjecture could be proved. More recently, stability has been recognized as a key condition to produce compactness results in Symplectic Field Theory [4, 8, 11] and Rabinowitz Floer homology [7]. This paper is motivated by the desire to generalize a result in the latter reference, as we explain below.

Let $F$ be any vector field spanning ker $\omega$. We say that $(\Sigma, \omega)$ is an Anosov Hamiltonian structure if the flow $\phi_{t}$ of $F$ is Anosov. Recall that this asserts the existence of a $d \phi_{t}$-invariant splitting

$$
T \Sigma=\mathbb{R} F \oplus E^{s} \oplus E^{u},
$$

where $\mathbb{R} F$ is the 1-dimensional distribution spanned by $F$, and such that there exist constants $C, \mu>0$ such that for all $x \in \Sigma$ and $t \geq 0$,

$$
\begin{gathered}
\left|d_{x} \phi_{t}(\xi)\right| \leq C|\xi| e^{-\mu t} \text { for } \xi \in E^{s}(x) ; \\
\left|d_{x} \phi_{-t}(\xi)\right| \leq C|\xi| e^{-\mu t} \text { for } \xi \in E^{u}(x) .
\end{gathered}
$$

The Anosov condition is invariant under time changes, and so is independent of the choice of vector field $F$. In other words, it is intrinsic to the Hamiltonian structure $(\Sigma, \omega)$. The weak bundles $E^{+}:=\mathbb{R} F \oplus E^{s}$ and $E^{-}:=\mathbb{R} F \oplus E^{u}$ are also invariant under time changes. 
We say that $\phi_{t}: \Sigma \rightarrow \Sigma$ is 1/2-pinched (or 1-bunched [20]) if there exist positive constants $C, A, a$ with $A<2 a$ such that

$$
\begin{gathered}
\frac{1}{C}|\xi| e^{-A t} \leq\left|d_{x} \phi_{t}(\xi)\right| \leq C|\xi| e^{-a t} \text { for } \xi \in E^{s} \text { and } t \geq 0, \\
\frac{1}{C}|\xi| e^{-A t} \leq\left|d_{x} \phi_{-t}(\xi)\right| \leq C|\xi| e^{-a t} \text { for } \xi \in E^{u} \text { and } t \geq 0 .
\end{gathered}
$$

We say that an Anosov Hamiltonian structure satisfies the 1/2-pinching condition if there exists some vector field $F$ spanning ker $\omega$ whose flow satisfies the 1/2-pinching condition.

Here is the situation we are actually interested in. Let $(M, g)$ be a closed Riemannian manifold that admits a background metric of negative curvature (possibly different from $g$ ) and $\pi: T M \rightarrow M$ the tangent bundle. Throughout the paper we let $\omega_{0}$ denote the symplectic form on $T M$ obtained by pulling back the canonical symplectic form $d x \wedge d p$ on $T^{*} M$ via the Riemannian metric. The form $\omega_{0}$ is exact; if $\alpha \in \Omega^{1}(T M)$ denotes the 1 -form defined by

$$
\alpha_{v}(\xi)=\left\langle d_{v} \pi(\xi), v\right\rangle
$$

then it is well known that $\omega_{0}=-d \alpha$. Suppose $\sigma \in \Omega^{2}(M)$ is a closed 2-form on $M$. Given $\varepsilon \in \mathbb{R}$ we define

$$
\omega_{\varepsilon}:=\omega+\varepsilon \pi^{*} \sigma
$$

Let $F_{\varepsilon}$ denote the symplectic gradient of the Hamiltonian

$$
H(x, v)=\frac{1}{2}|v|^{2}
$$

with respect to $\omega_{\varepsilon}$ and let $\phi_{t}^{\varepsilon}$ denote the flow of $F_{\varepsilon}$ with respect to $\omega_{\varepsilon}$. Note that $\phi_{t}^{0}$ is simply the geodesic flow. This flow models the motion of a particle of unit mass and charge $\varepsilon$ under the effect of a magnetic field, whose Lorentz force $Y: T M \rightarrow T M$ is the bundle map uniquely determined by

$$
\sigma_{x}(u, v)=\left\langle Y_{x}(u), v\right\rangle
$$

for all $u, v \in T_{x} M$ and all $x \in M$.

The family $\left\{\omega_{\varepsilon}\right\}$ for $\varepsilon \in[0,1]$ interpolates between the standard symplectic form $\omega_{0}$ and the form $\omega_{1}$. The form $\omega_{1}$ is called a twisted symplectic structure [2] and the flow $\phi_{t}^{1}$ is called a twisted geodesic flow or a magnetic flow.

Let $\Sigma_{k}=H^{-1}(k)$. We are interested in Anosov Hamiltonian structures of the form $\left(\Sigma_{k}, \omega_{1}\right)$.

Here is the main result we present. Let $n=\operatorname{dim} M$.

Theorem A. Suppose $\left(\Sigma_{k}, \omega_{1}\right)$ is an Anosov Hamiltonian structure and $n \geq 3$. Assume in addition:

- If $n$ is odd, $\left(\Sigma_{k}, \omega_{1}\right)$ has weak bundles of class $C^{1}$;

- If $n$ is even, $\left(\Sigma_{k}, \omega_{1}\right)$ is $1 / 2$-pinched.

Then $\left(\Sigma_{k}, \omega_{1}\right)$ is stable if and only if it is of contact type. In particular, if $\left(\Sigma_{k}, \omega_{1}\right)$ is stable, then $\sigma$ must be exact.

The last statement in the theorem can be seen as follows. Since $n=\operatorname{dim} M \geq 3$, the Gysin sequence of the sphere bundle $\pi \mid \Sigma_{k}: \Sigma_{k} \rightarrow M$ shows that $\left(\pi \mid \Sigma_{k}\right)^{*}$ : $H^{2}(M, \mathbb{R}) \rightarrow H^{2}\left(\Sigma_{k}, \mathbb{R}\right)$ is an isomorphism for $n \geq 4$ and injective for $n=3$. Since $\omega_{1}=-d \alpha+\pi^{*} \sigma$, the assertion that $\omega_{1}$ is exact on $\Sigma_{k}$ implies that $\pi^{*} \sigma \mid \Sigma_{k}$ is exact. Putting this together we conclude that $\sigma$ is exact. 
The bunching condition is a necessary one in the even dimensional case. Indeed, consider the twisted geodesic flow $\phi_{t}^{1}$ on compact quotients of complex hyperbolic space with $\sigma$ given by the Kähler form. Then for $k$ sufficiently large, $\phi_{t}^{1} \mid \Sigma_{k}$ is Anosov, and $\Sigma_{k}$ is stable but not contact ( $\sigma$ is not exact). The flow $\phi_{t}^{1}$ is algebraic and thus the stable and unstable bundles are real analytic. A stabilizing 1-form $\lambda$ can be defined by setting $\lambda\left(F_{1}\right)=1$ and ker $\lambda=E_{1}^{s} \oplus E_{1}^{u}$. The flow $\phi_{t}^{1}$ is not $1 / 2$-pinched since it has $2: 1$ resonances. It seems a reasonable conjecture that these are in fact the only cases where $C^{1}$ weak bundles is not sufficient to ensure that the conclusion of Theorem A holds. A well known theorem [21] states that the 1/2-pinching condition implies that the weak bundles are of class $C^{1}$. However the pinching condition is strictly stronger than requiring the weak bundles to be of class $C^{1}$ as the example of complex hyperbolic space described above shows.

We will prove in Proposition 5.11 that if $g$ is negatively curved and strictly $1 / 4$ pinched, for $k$ sufficiently large, the flow $\phi_{t}^{1}: \Sigma_{k} \rightarrow \Sigma_{k}$ is Anosov and 1/2-pinched. Thus, as a corollary of Theorem A we obtain the following.

Corollary B. Suppose $n \geq 3$ and $g$ is negatively curved and strictly 1/4-pinched. Then for any $k$ sufficiently large, the hypersurface $\Sigma_{k} \subset T M$ is not stable if $\sigma$ is not exact.

The corollary was first proved in [7, Theorem 1.4] for the case of $n$ even (but not Theorem A) and this previous result was the motivation for the present paper. It shows that the stability condition may fail for whole intervals of energy levels.

A caveat about the word "stable" is in order. One of the most remarkable features about Anosov systems is that they are structurally stable. This means that nearby systems are orbitally equivalent via a homeomorphism which in general is not $C^{1}$. The stability condition in Symplectic Geometry is equivalent to the existence of a thickening of the hypersurface with smoothly conjugate characteristic foliations 8 , Lemma 2.3]. In some sense it is this additional smoothness that is being exploited in Theorem A. One can put this into different words as follows: The existence of the form 1-form $\lambda$ means that one can find a parametrization of the characteristic foliation such that the hyperplane bundle $E^{s} \oplus E^{u}$ is smooth. In general, $E^{s} \oplus E^{u}$ is only Hölder continuous.

The assumption that $M$ admits a background metric of negative curvature is most likely superfluous. We use it to construct a conjugacy between our flow and the geodesic flow of a metric of negative curvature on $M$. We use this conjugacy to show that the fundamental group $\pi_{1}(M)$ acts as a 'North-South dynamics' (see Section 4 and in particular Theorem 4.2) on the space of stable leaves on the universal covering, and that the space of leaves admit a 'flip map' (see the discussion before the proof of Theorem (5.6). In order to remove the assumption of negative curvature one would need to prove Theorem 4.2 and Theorem 5.6 directly instead of constructing such a conjugacy.

Acknowlegment: We thank the referee for Remark 3.6.

\section{Preliminaries on Hamiltonian structures, holonomy and the Kanai CONNECTION}

Definition 2.1. A Hamiltonian structure is a pair $(\Sigma, \omega)$ where $\Sigma^{2 n-1}$ is a closed oriented manifold and $\omega$ is a closed 2 -form on $\Sigma$ such that $\omega^{n-1} \neq 0$ everywhere.

If $(\Sigma, \omega)$ is a Hamiltonian structure then ker $\omega$ defines an orientable 1-dimensional foliation of $\Sigma$, which we call the characteristic foliation. 
Definition 2.2. Let $\phi_{t}: N \rightarrow N$ be a smooth flow on a closed Riemannian manifold $N$, and let $F$ denote its infinitesimal generator. We say that $\phi_{t}$ is Anosov if there exists a $d \phi_{t}$-invariant splitting

$$
T \Sigma=\mathbb{R} F \oplus E^{s} \oplus E^{u},
$$

where $\mathbb{R} F$ is the 1-dimensional distribution spanned by $F$, and such that there exist constants $C, \mu>0$ such that for all $x \in \Sigma$ and $t \geq 0$,

$$
\begin{gathered}
\left|d_{x} \phi_{t}(\xi)\right| \leq C|\xi| e^{-\mu t} \text { for } \xi \in E^{s}(x) ; \\
\left|d_{x} \phi_{-t}(\xi)\right| \leq C|\xi| e^{-\mu t} \text { for } \xi \in E^{u}(x) .
\end{gathered}
$$

The Anosov property is invariant under time changes (see [10, Lemma 1.2] or [25. Proposition 17.4.5]), and hence we can define a Hamiltonian structure $(\Sigma, \omega)$ to be Anosov if the flow $\phi_{t}$ of any vector field $F$ spanning ker $\omega$ is Anosov.

We say that $\phi_{t}: \Sigma \rightarrow \Sigma$ is 1/2-pinched (or 1-bunched [20]) if there exist positive constants $C, A, a$ with $A<2 a$ such that

$$
\begin{gathered}
\frac{1}{C}|\xi| e^{-A t} \leq\left|d_{x} \phi_{t}(\xi)\right| \leq C|\xi| e^{-a t} \text { for } \xi \in E^{s} \text { and } t \geq 0, \\
\frac{1}{C}|\xi| e^{-A t} \leq\left|d_{x} \phi_{-t}(\xi)\right| \leq C|\xi| e^{-a t} \text { for } \xi \in E^{u} \text { and } t \geq 0 .
\end{gathered}
$$

We say that an Anosov Hamiltonian structure satisfies the 1/2-pinching condition if there exists some vector field $F$ spanning ker $\omega$ whose flow satisfies the 1/2-pinching condition.

The 1/2-pinching condition is a natural one to study, and should be thought of as a statement about being 'strongly hyperbolic'. Unsurprisingly, an Anosov system possessing this enhanced degree of hyperbolicity enjoys greater regularity. More specifically, write $E^{+}$and $E^{-}$for the weak stable and weak unstable bundles $E^{s} \oplus \mathbb{R} F$ and $E^{u} \oplus \mathbb{R} F$ respectively. If an Anosov Hamiltonian structure is $1 / 2$ pinched, then $E^{+}$and $E^{-}$are of class $C^{1}$ 21]. The next example will be crucial for the proof of Corollary B.

Example 2.3. Let $(M, g)$ be a closed manifold with negative sectional curvature $K$. Then the geodesic flow $\phi_{t}: S M \rightarrow S M$ is Anosov (see [25, Section 17.6]). In fact, we can say more. By compactness we can find constants $k_{1} \geq k_{0}>0$ such that

$$
-k_{1}^{2} \leq K \leq-k_{0}^{2}
$$

Then, comparison theorems show that [26, Theorem 3.2.17] (see also [27, Proposition 3.2]) there is a constant $C>0$ such that

$$
\begin{gathered}
\frac{1}{C}|\xi| e^{-k_{1} t} \leq\left|d_{v} \phi_{t}(\xi)\right| \leq C|\xi| e^{-k_{0} t} \text { for } \xi \in E^{s}(v) \text { and } t \geq 0 \\
\frac{1}{C}|\xi| e^{-k_{1} t} \leq\left|d_{v} \phi_{-t}(\xi)\right| \leq C|\xi| e^{-k_{0} t} \text { for } \xi \in E^{u}(v) \text { and } t \geq 0 .
\end{gathered}
$$

We see that $\phi_{t}$ is $1 / 2$-pinched as long as $k_{1}<2 k_{0}$. Therefore the geodesic flow of a metric whose sectional curvature satisfies $-4 \leq K<-1$ is $1 / 2$-pinched.

We return to two more definitions regarding Hamiltonian structures.

Definition 2.4. Let $(\Sigma, \omega)$ denote a Hamiltonian structure. We say that $(\Sigma, \omega)$ is stable if there exists a 1 -form $\lambda$ such that ker $\omega \subseteq$ ker $d \lambda$ and $\lambda \wedge \omega^{n-1}>0 . \lambda$ is called a stabilizing 1 -form. Note that if $\lambda$ is a stabilizing 1-form and $F$ is any vector field tangent to $\operatorname{ker} \omega$ we have $i_{F} d \lambda=0$. If $F$ is normalized so that $\lambda(F)=1$ then we say $F$ is the Reeb vector field of $\lambda$; note that the Reeb vector field is unique. 
A stronger condition is the following.

Definition 2.5. Let $(\Sigma, \omega)$ denote a Hamiltonian structure. We say that $(\Sigma, \omega)$ is of contact type if there exists a 1 -form $\lambda$ such that $d \lambda=\omega$ and $\lambda \wedge \omega^{n-1}>0$. Note if $(\Sigma, \omega)$ is of contact type then it is certainly stable, and that if $(\Sigma, \omega)$ is of contact type then $\omega$ is exact.

Now let $(\Sigma, \omega)$ denote a stable Anosov Hamiltonian structure with weak bundles of class $C^{1}$. Let $\lambda$ be a stabilizing 1 -form and let $F$ be the Reeb vector field of $\lambda$. Let $\phi_{t}$ denote the flow of $F$; then $\phi_{t}$ is Anosov and $T \Sigma=\mathbb{R} F \oplus E^{s} \oplus E^{u}$, with $\operatorname{ker} \lambda=E^{s} \oplus E^{u}$. Then the weak bundles are $C^{1}$; since $\lambda$ is $C^{\infty}$ (and so the bundle ker $\lambda$ is of class $C^{\infty}$ ), it follows that the strong bundles $E^{s}$ and $E^{u}$ are also of class $C^{1}$. The importance of this is that, as we shall see in Theorem 2.6 below, under these conditions there exists a unique connection $\nabla$ on $\Sigma$ called the Kanai connection which satisfies certain desirable properties. This was originally introduced by Kanai in [23]; see also [24, 14].

Holonomy. We briefly recall the concept of holonomy transport along the weak (un)stable foliations defined by an Anosov flow.

Throughout the remainder of this section, let $N$ denote a closed manifold of dimension $2 n-1$ and $\phi_{t}: N \rightarrow N$ an Anosov flow on $N$ with infinitesimal generator $F$. We also assume that $E:=E^{s} \oplus E^{u}$ is smooth and admits a smooth symplectic form $\omega$ which is $\phi_{t}$-invariant. We extend this form $\omega$ to a form defined on all of $T N$ by requiring that $i_{F} \omega=0$. As above $\lambda$ is the 1 -form defined by ker $\lambda=E$ and $\lambda(F)=1$.

It is well known that the subbundles $E^{s}$ and $E^{u}$, together with the weak bundles $E^{+}$and $E^{-}$, are all integrable. Namely, given any $x \in N$, we define

$$
W^{s}(x):=\left\{y \in N: \operatorname{dist}\left(\phi_{t} x, \phi_{t} y\right) \rightarrow 0 \text { as } t \rightarrow \infty\right\}
$$

and

$$
W^{u}(x):=\left\{y \in N: \operatorname{dist}\left(\phi_{t} x, \phi_{t} y\right) \rightarrow 0 \text { as } t \rightarrow-\infty\right\} .
$$

The sets $W^{s}(x)$ and $W^{u}(x)$ are injectively immersed manifolds called the strong (un) stable manifolds at $x$ and satisfy

$$
T_{x} W^{s}(x)=E^{s}(x), \quad T_{x} W^{u}(x)=E^{u}(x) .
$$

These define foliations $\mathcal{W}^{s}$ and $\mathcal{W}^{u}$ of $N$, called the strong (un)stable foliations. We assume throughout this section that these foliations $\mathcal{W}^{s}, \mathcal{W}^{u}$ are of class $C^{1}$.

Similarly, given $x \in N$, we define

$$
W^{+}(x):=\bigcup_{t \in \mathbb{R}} \phi_{t}\left[W^{s}(x)\right]=\bigcup_{t \in \mathbb{R}} W^{s}\left(\phi_{t} x\right)
$$

and

$$
W^{-}(x):=\bigcup_{t \in \mathbb{R}} \phi_{t}\left[W^{u}(x)\right]=\bigcup_{t \in \mathbb{R}} W^{u}\left(\phi_{t} x\right),
$$

which are then the weak (un) stable manifolds at $x$. They satisfy

$$
T_{x} W^{+}(x)=E^{+}(x), \quad T_{x} W^{-}(x)=E^{-}(x),
$$

and define foliations $\mathcal{W}^{+}$and $\mathcal{W}^{-}$of $M$.

Consider the foliations $\mathcal{W}^{u}$ and $\mathcal{W}^{+}$. It is well known that these are transverse to each other (i.e. $E^{u}(x) \cap E^{+}(x)=\{0\}$ ) and have complementary dimensions $n-1$ and $n$ respectively. The same is of course true of the foliations $\mathcal{W}^{s}$ and $\mathcal{W}^{-}$, and all of what we say below can be repeated for them.

By a foliation chart we mean a diffeomorphism $\varphi: U \rightarrow(-1,1)^{n-1} \times(-1,1)^{n}$ of the form $x \mapsto\left(\varphi_{1}(x), \varphi_{2}(x)\right)$ where $U \subseteq N$ is open, such that the connected 
components of $\mathcal{W}^{u} \mid U$ are given by $\varphi_{2}=$ const and the connected components of $\mathcal{W}^{+} \mid U$ are given by $\varphi_{1}=$ const. We then call $U$ a foliated neighborhood.

Let $(\varphi, U)$ denote a foliated chart defined on $N$. Given $x \in U$, let $W_{U}^{u}(x):=$ $W^{u}(x) \cap U$. Suppose $y \in W_{U}^{+}(x)$ lies in the same connected component of $W_{U}^{+}(x)$ as $x$. We want to define the holonomy map $\mathcal{H}_{x, y}^{U}: W_{U}^{u}(x) \rightarrow W_{U}^{u}(y)$ along the leaves of $\mathcal{W}^{+}$. This is defined as follows. Suppose $p \in W_{U}^{u}(x)$. Then there exists a unique point $q \in W^{+}(p) \cap W_{U}^{u}(y)$, and we define $\mathcal{H}_{x, y}^{U}(p)=q$. The map $\mathcal{H}_{x, y}^{U}$ is of class $C^{r}$ for $r \geq 1$ if the foliations $\mathcal{W}^{u}$ and $\mathcal{W}^{+}$are also of class $C^{r}$.

More generally, suppose $\gamma:[0, T] \rightarrow N$ is a smooth curve such that $\gamma(0)=x$ and $\gamma(t) \in W^{+}(x)$ for all $t$. Let $y:=\gamma(T) \in W^{+}(x)$. Then we can define $\mathcal{H}_{x, y}^{\gamma}$ by covering the image of $\gamma$ with foliated charts $\left(\varphi_{i}, U_{i}\right)$ for $i=1, \ldots, l$ with $x \in U_{1}$ and $y \in U_{l}$, and choosing points $0=t_{0}, \ldots, t_{l}=T$ such that $\gamma\left(t_{i-1}\right), \gamma\left(t_{i}\right) \in U_{i}$ for each $1 \leq i \leq l$ and then setting

$$
\mathcal{H}_{x, y}^{\gamma}:=\mathcal{H}_{\gamma\left(t_{l-1}\right), y}^{U_{l}} \circ \mathcal{H}_{\gamma\left(t_{l-2}\right), \gamma\left(t_{l-1}\right)}^{U_{l-1}} \circ \cdots \circ \mathcal{H}_{x, \gamma\left(t_{1}\right)}^{U_{1}} .
$$

It can be shown that $\mathcal{H}_{x, y}^{\gamma}$ only depends on the homotopy class of $\gamma \in W^{+}(x)$. One can check that if $x, y, z$ are in the image of $\gamma$, then after suitably restricting the domains of definition, it holds that

$$
\mathcal{H}_{y, z}^{\gamma} \circ \mathcal{H}_{x, y}^{\gamma}=\mathcal{H}_{x, z}^{\gamma} \text {. }
$$

Moreover, since the foliations are $\phi_{t}$-invariant, for any curve $\gamma$ in the weak unstable foliation $\mathcal{W}^{+}$we have

$$
\phi_{t} \circ \mathcal{H}_{x, y}^{\gamma} \circ \phi_{-t}=\mathcal{H}_{\phi_{t} x, \phi_{t} y}^{\phi_{t} \circ \gamma} .
$$

Next, we consider the differential of $\mathcal{H}_{x, y}^{\gamma}$, known as the holonomy transport along $\gamma$

$$
H_{x, y}^{\gamma}(p):=d_{p} \mathcal{H}_{x, y}^{\gamma}: T_{p} W^{u}(x) \rightarrow T_{\mathcal{H}_{x, y}^{\gamma}(p)} W^{u}(y) .
$$

In particular we write

$$
H_{x, y}^{\gamma}: E^{u}(x) \rightarrow E^{u}(y)
$$

for the map $H_{x, y}^{\gamma}(x): T_{x} W^{u}(x) \rightarrow T_{y} W^{u}(y)$. Note that differentiating (2.3) gives

$$
d_{y} \phi_{t} \circ H_{x, y}^{\gamma} \circ d_{\phi_{-t} x} \phi_{-t}=H_{\phi_{t} x, \phi_{t} y}^{\phi_{t} \circ \gamma} \quad \text { as maps } E^{u}\left(\phi_{t} x\right) \rightarrow E^{u}\left(\phi_{t} y\right) .
$$

We say that a vector field $X \in \Gamma\left(E^{u}\right)$ is invariant under the holonomy transport along the leaves of $\mathcal{W}^{+}$if for any curve $\gamma$ from $x$ to $y$ contained in $W^{+}(x)$ it holds that

$$
H_{x, y}^{\gamma}(p)(X(p))=X\left(\mathcal{H}_{x, y}^{\gamma}(p)\right) \quad \text { for all } p \in W^{u}(x) .
$$

The Kanai connection. We now recall the definition and main features of the Kanai connection.

Let $I$ be the $(1,1)$-tensor on $N$ given by $I(v)=-v$ for $v \in E^{s}, I(v)=v$ for $v \in E^{u}$ and $I(F)=0$. Consider the symmetric non-degenerate bilinear form given by

$$
h(X, Y):=\omega(X, I Y)+\lambda \otimes \lambda(X, Y) .
$$

The pseudo-Riemannian metric $h$ is of class $C^{1}$ and thus there exists a unique $C^{0}$ affine connection $\nabla$ such that:

(1) $h$ is parallel with respect to $\nabla$;

(2) $\nabla$ has torsion $\omega \otimes F$.

Theorem 2.6. The Kanai connection $\nabla$ has the following properties:

(1) $\nabla$ is $\phi_{t}$-invariant, $\nabla \omega=0, \nabla_{F}=L_{F}$ and $\nabla F=0$. 
(2) The Anosov splitting is invariant under $\nabla$, that is, if $X_{s} \in \Gamma\left(E^{s}\right), X_{u} \in$ $\Gamma\left(E^{u}\right)$ and $Y$ is any vector field on $N$ then

$$
\nabla_{Y} X_{s} \in \Gamma\left(E^{s}\right), \quad \nabla_{Y} X_{u} \in \Gamma\left(E^{u}\right) .
$$

(3) The restriction of $\nabla$ to each leaf of the foliations $\mathcal{W}^{s}$ and $\mathcal{W}^{u}$ of $N$ is flat (note that restriction of the connection to the leaves of the stable and unstable foliations is smooth so it makes sense to talk about its curvature).

(4) Parallel transport along curves on the weak stable and unstable manifolds coincide with the holonomy transport determined by the stable and unstable foliations.

Remark 2.7. Let us observe that since we know that the restriction of $\nabla$ to each leaf of $\mathcal{W}^{s}$ and $\mathcal{W}^{u}$ is flat, it follows that $H_{x, y}^{\gamma}$ is independent of $\gamma$. Thus we will omit $\gamma$ from the notation and simply write $H_{x, y}$.

Lemma 2.8. The holonomy transport $H_{x, \phi_{t} x}$ is given by $d_{x} \phi_{t} \mid E^{u}(x)$, that is,

$$
H_{x, \phi_{t} x}=d_{x} \phi_{t} \mid E^{u}(x) \text { as maps } E^{u}(x) \rightarrow E^{u}\left(\phi_{t} x\right) .
$$

Proof. Fix $x \in N$, and let $\Gamma(t):=\phi_{t} x$. Fix $\xi \in E^{u}(x)$, and let $V(t)$ denote the vector field along $\Gamma(t)$ defined by $V(t)=d_{x} \phi_{t}(\xi)$. It suffices to show that $V$ is parallel: $\nabla_{\dot{\Gamma}} V \equiv 0$.

Note that $\dot{\Gamma}(t)=F\left(\phi_{t} x\right)$. Since $\nabla_{F}=L_{F}$ we have

$$
\nabla_{F\left(\phi_{t} x\right)} V(t)=\left.\frac{d}{d t}\right|_{t=0} d_{\phi_{t} x} \phi_{-t}\left(V\left(\phi_{t} x\right)\right)=\left.\frac{d}{d t}\right|_{t=0} \xi=0 .
$$

and the lemma follows.

\section{Constructing the Invariant Subbundles}

Throughout this section let $(\Sigma, \omega)$ denote a stable Anosov Hamiltonian structure of dimension $2 n-1$ where $n \geq 2, \lambda$ a stabilizing 1 -form for $(\Sigma, \omega), F$ the Reeb vector field of $\lambda$, and $\phi_{t}$ the flow of $F$. If $n$ is odd, suppose that the weak (un)stable bundles are of class $C^{1}$. If $n$ is even, assume that the Anosov Hamiltonian structure is $1 / 2$ pinched. In either case, the Kanai connection $\nabla$ is defined. The goal in this section is to construct a subbundle of $E^{u}$ that is invariant under both $\phi_{t}$ and the holonomy transport along the leaves of the weak stable foliation $\mathcal{W}^{+}$. It is the existence of this subbundle that we will then exploit in Section 5 in order to prove Theorem A from the introduction. The main ideas for these constructions come from [19, 13, 14, 24].

In the even dimensional case we will need to know that $d \lambda$ is parallel with respect to the Kanai connection. This is the only place in the paper where we will actually use the 1/2-pinching condition (as opposed to just $C^{1}$ weak (un)stable bundles). The following lemma is due to Kanai (24, Lemma 3.2]).

Lemma 3.1. Suppose $\phi_{t}$ is a time change of a 1/2-pinched Anosov flow. Then $\nabla(d \lambda)=0$.

Proof. Suppose $\tau$ is any invariant $(0,3)$-tensor annihilated by $F$, i.e. $i_{F} \tau=0$. We claim that $\tau$ must vanish. Note that if $\psi_{t}$ is any time change of $\phi_{t}$, then $\psi_{t}$ also leaves $\tau$ invariant since $F$ annihilates $\tau$, so in the proof below without loss of generality, we may assume that $\phi_{t}$ itself is $1 / 2$-pinched.

To see that $\tau$ vanishes consider for example a triple of vectors $\left(\xi_{1}, \xi_{2}, \xi_{3}\right)$ where $\xi_{1}, \xi_{2} \in E^{s}(x)$ but $\xi_{3} \in E^{u}(x)$. Then there is a constant $C>0$ such that

$$
\begin{aligned}
\left|\tau_{x}\left(\xi_{1}, \xi_{2}, \xi_{3}\right)\right| & =\left|\tau_{\phi_{t} x}\left(d_{x} \phi_{t}\left(\xi_{1}\right), d_{x} \phi_{t}\left(\xi_{2}\right), d_{x} \phi_{t}\left(\xi_{3}\right)\right)\right| \\
& \leq C e^{(A-2 a) t}\left|\xi_{1}\right|\left|\xi_{2} \| \xi_{3}\right|
\end{aligned}
$$


By the 1/2-pinching condition the last expression tends to zero as $t \rightarrow \infty$ and therefore $\tau_{x}\left(\xi_{1}, \xi_{2}, \xi_{3}\right)=0$. The same will happen for other possible triples $\left(\xi_{1}, \xi_{2}, \xi_{3}\right)$ when we let $t \rightarrow \pm \infty$.

Since $d \lambda$ and $\nabla$ are $\phi_{t}$-invariant, so is $\nabla(d \lambda)$. Since $i_{F} d \lambda=0, \nabla(d \lambda)$ is also annihilated by $F$ (to see that $\nabla_{F}(d \lambda)=0$ use that $d \lambda$ is $\phi_{t}$-invariant and that $\left.\nabla_{F}=L_{F}\right)$. Hence by the previous argument applied to $\tau=\nabla(d \lambda)$ we conclude that $\nabla(d \lambda)=0$ as desired.

Lemma 3.2. There exists a smooth $\phi_{t}$-invariant bundle map $L: E \rightarrow E$ such that for $X, Y \in \Gamma(E)$,

$$
d \lambda(X, Y)=\omega(L X, Y)=\omega(X, L Y) .
$$

Moreover $L$ preserves the decomposition of $E=E^{s} \oplus E^{u}$, that is, $L=L^{s}+L^{u}$ where $L^{s}: E^{s} \rightarrow E^{s}$ and $L^{u}: E^{u} \rightarrow E^{u}$.

Proof. Since $d \lambda$ is $\phi_{t}$-invariant and annihilated by $F$, there exists a $\phi_{t}$-invariant smooth section $L$ of $E^{*} \otimes E$ such that the stated equation holds. It remains to check that $L$ preserves the decomposition, that is, $L$ commutes with $I$. But this is clear: if $X_{s}, Y_{s} \in \Gamma\left(E^{s}\right)$ then

$$
d \lambda\left(X_{s}, Y_{s}\right)=X_{s} \lambda\left(Y_{s}\right)-Y_{s} \lambda\left(X_{s}\right)-\lambda\left(\left[X_{s}, Y_{s}\right]\right)
$$

using integrability of $E^{s}$ and the fact that $\operatorname{ker} \lambda=E$, we see that

$$
0=d \lambda\left(X_{s}, Y_{s}\right)=\omega\left(L X_{s}, Y_{s}\right),
$$

and hence $L X_{s} \in \Gamma\left(E^{s}\right)$. The same argument applies with sections of $E^{u}$.

The construction of the invariant subbundle will depend on the parity of $n$. We will begin with the easier case, when $n$ is even.

The case of $n$ even. Since $\operatorname{dim} \Sigma=2 n-1$ and $n$ is even, we have $\operatorname{dim} E^{u}=n-1$ an odd number. Thus for any $x \in \Sigma$, the map $L_{x}^{u}: E^{u}(x) \rightarrow E^{u}(x)$ admits a real eigenvalue $\rho_{x}$. In fact, we have the following result.

Lemma 3.3. There exists $\rho \in \mathbb{R}$ such that $\rho_{x}=\rho$ for all $x \in \Sigma$.

Remark 3.4. In the proof of the lemma we will make use of the fact that $\phi_{t}$ is a transitive flow. To see this, we first note that since $\phi_{t}$ preserves a probability measure, the non-wandering set $\Omega(\phi)$ of $\phi_{t}$ is necessarily equal to all of $\Sigma$ (see for instance [1] or [25, Chapter 18]). It is then a standard result that an Anosov flow whose non-wandering set is the whole space is transitive (see for instance [25, p.576]). We also remark that since $\phi_{t}$ is Anosov the set of periodic points is dense in the non-wandering set $\Omega(\phi)$, and hence dense in $\Sigma$; we will use this observation in the next subsection.

Proof. For $k \geq 0$, let $a_{k}(x)$ denote the coefficient of $t^{k}$ in the characteristic polynomial $p_{x}(t)$ of $L_{x}^{u}$. Then $a_{k}: \Sigma \rightarrow \mathbb{R}$ is continuous and $\phi_{t}$-invariant. Since $\phi_{t}$ is transitive, $a_{k}$ is constant. Thus the characteristic polynomial $p_{x}(t)$ of $L_{x}^{u}$ is independent of $x$, and so each $L_{x}^{u}$ admits the same eigenvalues.

Let therefore $\rho_{0} \in \mathbb{R}$ be a common eigenvalue of the maps $\left\{L_{x}^{u}\right\}$. Let

$$
P_{\rho_{0}}(x):=\left\{\xi \in E^{u}(x): L_{x}^{u} \xi=\rho_{0} \xi\right\} \neq\{0\} .
$$

Proposition 3.5. The map $x \mapsto P_{\rho_{0}}(x)$ defines a $C^{1}$ subbundle of $E^{u}$. Moreover for $x \in \Sigma$, the restriction of $P_{\rho_{0}}$ to $W^{u}(x)$ is integrable. 
Proof. Since $d \lambda$ is $\nabla$-parallel by Lemma 3.1, $P_{\rho_{0}}(x)$ is invariant under the parallel transport of $\nabla$. More precisely, given a curve $\gamma$ from $x$ to $y$, if $\mathcal{P}_{x, y}^{\gamma}: T_{x} \Sigma \rightarrow T_{y} \Sigma$ denotes the parallel transport along $\gamma$ with respect to $\nabla$, then

$$
\mathcal{P}_{x, y}^{\gamma} P_{\rho_{0}}(x) \subseteq P_{\rho_{0}}(y),
$$

since for $\xi \in E^{u}(x)$, if $L_{x}^{u} \xi=\rho_{0} \xi$ then

$$
L_{y}^{u} \mathcal{P}_{x, y}^{\gamma}(\xi)=\rho_{0} \mathcal{P}_{x, y}^{u}(\xi)
$$

Indeed, we have for any vector fields $X, Y, Z$ that:

$$
\begin{aligned}
0 & =\left(\nabla_{X} d \lambda\right)(Y, Z) \\
& =\nabla_{X}(d \lambda(Y, Z))-d \lambda\left(\nabla_{X} Y, Z\right)-d \lambda\left(Y, \nabla_{X} Z\right) \\
& =\nabla_{X}(\omega(L Y, Z))-\omega\left(L\left(\nabla_{X} Y\right), Z\right)-\omega\left(L Y, \nabla_{X} Z\right) \\
& =\nabla_{X}(\omega(L Y, Z))-\omega\left(\nabla_{X}(L Y), Z\right)-\omega\left(L Y, \nabla_{X} Z\right)+\omega\left(\left(\nabla_{X} L\right) Y, Z\right) \\
& =\nabla_{X} \omega(L Y, Z)+\omega\left(\left(\nabla_{X} L\right) Y, Z\right) \\
& =\omega\left(\left(\nabla_{X} L\right) Y, Z\right)
\end{aligned}
$$

where the last equality used the fact that $\nabla \omega=0$. Thus $L$ is parallel, and hence $L^{u}$ and $L^{s}$ are also parallel. Thus:

$$
\begin{aligned}
L_{y}^{u} \mathcal{P}_{x, y}^{\gamma}(\xi) & =\mathcal{P}_{x, y}^{\gamma}\left(L_{x}^{u} \xi\right) \\
& =\mathcal{P}_{x, y}^{\gamma}\left(\rho_{0} \xi\right) \\
& =\rho_{0} \mathcal{P}_{x, y}^{\gamma}(\xi) .
\end{aligned}
$$

It remains to check that the restriction of $P_{\rho_{0}}$ to $W^{u}(x)$ is integrable. Note that if $X$ and $Y$ are parallel sections of $E^{u}$ over $W^{u}(x)$ then since $\nabla$ has no torsion over $E^{u}$, we have

$$
0=\nabla_{X} Y-\nabla_{Y} X=[X, Y]
$$

The case of $n$ odd. We now want to construct a $\phi_{t}$-invariant subbundle $P_{\rho_{0}} \subseteq E^{u}$ that is invariant under holonomy transport along the leaves of $\mathcal{W}^{+}$for the case when $n$ is an odd integer. As before we construct the maps $L_{x}^{u}: E^{u}(x) \rightarrow E^{u}(x)$; however in this case since $\operatorname{dim} E^{u}(x)=n-1$ is even, it is no longer necessarily the case that $L_{x}^{u}$ admits a real eigenvalue, and so our previous construction will not work.

Remark 3.6. Recall we are only assuming that $\phi_{t}$ is a time change of a $1 / 2$-pinched Anosov flow in the even dimensional case. Thus in the odd dimensional case Lemma 3.1 is not available to us. If however we did assume that $\nabla(d \lambda)=0$ then we could dramatically simplify the treatment of the odd-dimensional case both in this section and in Section 5. Indeed, whilst in the odd-dimensional case the characteristic polynomial $p(t)$ of $L^{u}$ no longer necessarily admits a real eigenvalue, it is reducible over $\mathbb{R}$ to a product of quadratic factors, say $p(t)=q_{1}(t) \ldots q_{k}(t)$. We may then define sub-bundles $P_{i}:=\operatorname{ker} q_{i}\left(L^{u}\right)$ of $E^{u}$, which have constant non-zero dimension. Since $\nabla(d \lambda)=0$ these are parallel and hence integrable. We thank the referee for this observation.

Let $E_{\mathbb{C}}^{u}:=E^{u} \otimes \mathbb{C}$ denote the complexification of $E^{u}$, and let $\mathbb{L}: E_{\mathbb{C}}^{u} \rightarrow E_{\mathbb{C}}^{u}$ denote the complex linear extension of $L^{u}$. Given $\rho \in \mathbb{C}$, we set

$$
Q_{\rho}(x):=\left\{\xi \in E^{u}(x): \xi=\operatorname{Re} \zeta \text { for some } \zeta \in E_{\mathbb{C}}^{u} \text { with } \mathbb{L}_{x} \zeta=\rho \zeta\right\} .
$$

By the same arguments as those used in the proof of Lemma 3.3 , there exists an open $\phi_{t}$-invariant set $O \subseteq \Sigma$ with the property that for any fixed $\rho \in \mathbb{C}$, the dimension of the subspaces $Q_{\rho}(x)$ is constant for $x \in O$. Thus $Q_{\rho} \mid O$ defines a continuous $\phi_{t}$-invariant subbundle of $E^{u} \mid O$ for all $\rho \in \mathbb{C}$. 
Now define a new bundle $P_{\rho}$ over all of $\Sigma$ by setting

$$
P_{\rho}(x):=\bigcap\left\{H_{y, x} Q_{\rho}(y): y \in O \cap W^{s}(x)\right\} .
$$

We call $P_{\rho}$ the holonomy intersection of $Q_{\rho}$ (see [19, p685], as well as [13, Section $8]$ ). Note that $P_{\rho}$ is clearly holonomy invariant. The next result is Lemma 2.7 in [19].

Theorem 3.7. For all $\rho \in \mathbb{C}, P_{\rho}$ is a continuous $\phi_{t}$-invariant subbundle of $E^{u}$ over $\Sigma$.

Proof. Let

$$
K:=\min \left\{\operatorname{dim} P_{\rho}(x): x \in \Sigma\right\} .
$$

Since the dimension of $K$ is constant along the leaves of $\mathcal{W}^{s}$, there exists $x_{0} \in O$ with $\operatorname{dim} P_{\rho}\left(x_{0}\right)=K$. We can choose points $x_{1}, \ldots, x_{\ell} \in W^{s}\left(x_{0}\right) \cap O$ such that

$$
P_{\rho}\left(x_{0}\right)=\bigcap_{i=1}^{\ell} H_{x_{i}, x_{0}} Q_{\rho}\left(x_{i}\right) .
$$

Since $Q_{\rho}$ and the holonomy maps $H_{x_{i}, x_{0}}$ are continuous there exists an open neighborhood $O_{0} \subseteq O \cap W^{-}\left(x_{0}\right)$ of $x_{0}$ such that the following holds. Given $y_{0} \in O_{0}$ there exist open neighborhoods $U_{i}$ of $x_{i}$ such that the connected component of $x_{i}$ in the set $U_{i} \cap W^{s}\left(y_{0}\right) \cap W^{u}\left(x_{i}\right)$ consists of a single point $y_{i}$, and moreover that

$$
\operatorname{dim} \bigcap_{i=1}^{\ell} H_{y_{i}, y_{0}} Q_{\rho}\left(y_{0}\right) \leq K .
$$

By minimality of $K$ this forces

$$
P_{\rho}\left(y_{0}\right)=\bigcap_{i=1}^{\ell} H_{y_{i}, y_{0}} Q_{\rho}\left(y_{0}\right) ;
$$

in particular this proves we can find a neighborhood $V$ of $x_{0}$ in $\Sigma$ such that the restriction of $P_{\rho}$ to $V$ is a continuous subbundle of $E^{u} \mid V$. But then since

$$
\Sigma=\bigcup_{y \in V} W^{s}(y)
$$

it follows that $P_{\rho}$ is a continuous subbundle of $E^{u}$ over all of $\Sigma$.

Unfortunately, it is not necessarily the case that $P_{\rho}$ is of positive dimension. To ensure this, we will need to choose $\rho \in \mathbb{C}$ carefully. Here is the general idea; the precise construction (due to Hamenstädt [19, Section 2]) is somewhat technical. We choose a periodic point $q$ of $\phi_{t}$, with period $T>0$, say. Then $d_{q} \phi_{T}: T_{q} \Sigma \rightarrow T_{q} \Sigma$ induces a map $A_{q}^{u}: E^{u}(q) \rightarrow E^{u}(q)$. Let $\mathbb{A}_{q}$ denote the complex linear extension of $A_{q}^{u}$ to a map $E_{\mathbb{C}}^{u}(q) \rightarrow E_{\mathbb{C}}^{u}(q)$, and let $\sigma \in \mathbb{R}, \sigma>1$ denote the minimal absolute value of an eigenvalue of $\mathbb{A}_{q}$. We are interested in the subspace $S(q) \subseteq E^{u}(q)$ consisting of the subspace spanned by the union of the eigenspaces of $\mathbb{A}_{q}$ corresponding to eigenvalues of absolute value $\sigma$.

We then use holonomy transport to carry $S(q)$ to subspaces $S(x) \subseteq E^{u}(x)$ for $x \in W^{+}(q)$, thus creating a distribution $S$ over $E^{u} \mid W^{+}(q)$.

What is the point of this construction? Suppose now $q \in O$ (where $O$ is the open set defined earlier on which $Q_{\rho}$ is a continuous subbundle of $E^{u} \mid O$ for all $\rho \in \mathbb{C}$ such $q$ always exist since the set of periodic points of $\phi_{t}$ is dense in $\Sigma$, see Remark 3.4). Then one shows that $L_{q}^{u}: E^{u}(q) \rightarrow E^{u}(q)$ preserves the subbundle $S(q)$, and 
thus $\mathbb{L}_{q}: E_{\mathbb{C}}^{u}(q) \rightarrow E_{\mathbb{C}}^{u}(q)$ preserves the complexification $S_{\mathbb{C}}(q) \subseteq E_{\mathbb{C}}^{u}(q)$. Moreover if $\rho_{0}$ is an eigenvalue of $\mathbb{L}_{q} \mid S_{\mathbb{C}}(q)$, we will show that the subspace

$$
Q_{\rho_{0}}(q) \cap S(q) \subseteq E^{u}(q)
$$

(which is necessarily of positive dimension) is contained in $P_{\rho_{0}}(q)$; in other words, for this choice of $\rho_{0}, P_{\rho_{0}}$ is of positive dimension, and this gives us our desired subbundle of $E^{u}$ for the case where $n$ is odd.

We will now begin with the details of the construction.

Since $q$ is periodic of period $T, d_{q} \phi_{T}: T_{q} \Sigma \rightarrow T_{q} \Sigma$ defines a hyperbolic linear map $A_{q}: E(q) \rightarrow E(q)$ which preserves $E^{s}(q)$ and $E^{u}(q)$, and so defines maps $A_{q}^{s}: E^{s}(q) \rightarrow E^{s}(q)$ and $A_{q}^{u}: E^{u}(q) \rightarrow E^{u}(q)$. Let $\mathbb{A}_{q}: E_{\mathbb{C}}^{u}(q) \rightarrow E_{\mathbb{C}}^{u}(q)$ denote the linear map induced by $A_{q}^{u}$. Let $\sigma \in \mathbb{R}$ denote the smallest absolute value of an eigenvalue of $\mathbb{A}_{q}$ (note $\sigma>1$ as $d \phi_{t} \mid E^{u}$ is expanding).

Now let us set

$S(q):=\operatorname{span}\left\{\xi \in E^{u}(q): \exists \zeta \in E_{\mathbb{C}}^{u}(q), \rho \in \mathbb{C},|\rho|=\sigma\right.$, with $\left.\xi=\operatorname{Re} \zeta, \mathbb{A}_{q} \zeta=\rho \zeta\right\}$.

Then for $x \in W^{+}(q)$ we define

$$
S(x):=H_{q, x}[S(q)] .
$$

Then $S$ is a $C^{1}$-subbundle of $E^{u} \mid W^{+}(q)$ and moreover using (2.4) and Lemma 2.8 we see $d_{x} \phi_{t}[S(x)] \subseteq S\left(\phi_{t} x\right)$ for all $x \in W^{+}(q)$; in particular $A_{q}^{u}$ maps $S(x)$ to itself.

We now make a little digression into some elementary linear algebra. Given an invertible complex linear endomorphism $A: \mathbb{C}^{m} \rightarrow \mathbb{C}^{m}$, decompose $\mathbb{C}^{m}=\bigoplus_{j=1}^{k} V^{\rho_{j}}$ into the root spaces of $A$, and let $\left\{\xi_{1}^{\rho_{1}}, \ldots, \xi_{d\left(\rho_{1}\right)}^{\rho_{1}}, \xi_{1}^{\rho_{2}}, \ldots, \xi_{d\left(\rho_{2}\right)}^{\rho_{2}}, \ldots, \xi_{1}^{\rho_{k}}, \ldots, \xi_{d\left(\rho_{k}\right)}^{\rho_{k}}\right\}$ denote a basis of $\mathbb{C}^{m}$ such that $A$ is in Jordan normal form with respect to this basis. That is, we have $d\left(\rho_{j}\right)=\operatorname{dim} V^{\rho_{j}}, A \xi_{1}^{\rho_{j}}=\rho_{j} \xi_{1}^{\rho_{j}}$ for $j=1, \ldots, k$ and $A \xi_{i}^{\rho_{j}}=\xi_{1}^{\rho_{j}}+\xi_{2}^{\rho_{j}}+\cdots+\xi_{i-1}^{\rho_{j}}+\rho_{j} \xi_{i}^{\rho_{j}}$ for $i>1$ and $j=1, \ldots, k$. Now set

$$
\eta_{i}^{\rho_{j}}:=\frac{\rho_{j}}{\left|\rho_{j}\right|} \xi_{i}^{\rho_{j}},
$$

and define a Hermitian inner product $\langle\cdot, \cdot\rangle$ on $\mathbb{C}^{m}$ by declaring $\left\{\eta_{i}^{\rho_{j}}\right\}$ to be a unitary basis.

Let $\sigma:=\min \left\{\left|\rho_{j}\right|: j=1 \ldots, k\right\}$, and set

$$
S:=\operatorname{span}_{\mathbb{C}}\left\{\xi_{1}^{\rho_{j}}:\left|\rho^{j}\right|=\sigma\right\} .
$$

Then for any $\xi, \eta \in S$ we have

$$
\langle A \xi, A \eta\rangle=\sigma^{2}\langle\xi, \eta\rangle,
$$

The following result is essentially due to Hamenstädt.

Lemma 3.8. Let $\xi, \eta \in \mathbb{C}^{n}$, with $\eta \in S, \eta \neq 0$ and $\xi \notin S$. Then

$$
\lim _{k \rightarrow \infty} \frac{\left|A^{k} \xi\right|}{\left|A^{k} \eta\right|}=\infty \text {. }
$$

Proof. The crux of the proof is the following formula, whose proof can be found in [19, Corollary 2.3]. Suppose $\zeta \in \mathbb{C}^{n}$ is a root vector for $A$ with eigenvalue $\rho \neq 0$, that is, there exists $j \in \mathbb{N}$ such that

$$
(A-\rho \mathrm{Id})^{j} \zeta=0 \quad \text { but } \quad(A-\rho \mathrm{Id})^{j-1} \zeta \neq 0 .
$$

Then

$$
\lim _{k \rightarrow \infty} \frac{A^{k} \zeta}{\rho^{k} k^{j-1}}=\frac{1}{\rho^{j-1}(j-1) !}(A-\sigma \mathrm{Id})^{j-1} \zeta
$$


In particular, if either:

(1) $\rho, \rho^{\prime}$ are eigenvalues of $A$ with $|\rho|<\left|\rho^{\prime}\right|$ and $\zeta, \zeta^{\prime} \in \mathbb{C}^{N}$ are root vectors for $A$ with eigenvalues $\rho, \rho^{\prime}$ or

(2) $\zeta$ and $\zeta^{\prime}$ are both root vectors for $A$ with eigenvalue $\rho$, such that there exists $j \in \mathbb{N}$ with

$$
(A-\rho \mathrm{Id})^{j} \zeta=0 \quad \text { but } \quad(A-\rho \mathrm{Id})^{j} \zeta^{\prime} \neq 0,
$$

then

This implies the lemma.

$$
\lim _{k \rightarrow \infty} \frac{\left|A^{k} \zeta^{\prime}\right|}{\left|A^{k} \zeta\right|}=\infty
$$

We return now to the problem at hand and prove the following result ([19, Lemma 2.5]).

Proposition 3.9. The map $L^{u}$ preserves the bundle $S$ over $E^{u} \mid W^{+}(q)$.

Before getting started on the proof, we introduce an auxilliary inner product that will be helpful for this result and its sequel. Let us fix an inner product $\langle\cdot, \cdot\rangle_{q}$ on $E^{u}(q)$ with the property that if $\xi, \eta \in S(q)$ then $\left\langle A_{q}^{u} \xi, A_{q}^{u} \eta\right\rangle_{q}=\sigma^{2}\langle\xi, \eta\rangle_{q}$ (such an inner product exists by the discussion above). Then extend $\langle\cdot, \cdot\rangle_{q}$ to an inner product $\langle\cdot, \cdot\rangle_{x}$ for all $x \in W^{s}(q)$ by

$$
\langle\xi, \eta\rangle_{x}:=\left\langle H_{x, q}(\xi), H_{x, q}(\eta)\right\rangle_{q} \quad \text { for } x \in W^{s}(q), \xi, \eta \in E^{u}(x) .
$$

Let $|\cdot|_{x}$ denote the norm induced by $\langle\cdot, \cdot\rangle_{x}$ for $x \in W^{s}(q)$.

Proof. (of Proposition 3.9)

First note that by (2.4) for any $x \in W^{s}(q)$ and $k \in \mathbb{Z}$,

$$
H_{\phi_{k T} x, q} \circ d_{x} \phi_{k T}=\left(A_{q}^{s}\right)^{k} \circ H_{x, q} \text { as maps } E^{u}(x) \rightarrow E^{u}(q) \text {. }
$$

Thus if $\xi \in E^{u}(x), \xi \notin S(x)$ and $\eta \in S(x), \eta \neq 0$ we have

$$
\begin{aligned}
\frac{\left|d_{x} \phi_{k T}(\xi)\right|_{\phi_{k T} x}}{\left|d_{x} \phi_{k T}(\eta)\right|_{\phi_{k T} x}} & =\frac{\left|H_{\phi_{k T} x, q}\left(d_{x} \phi_{k T}(\xi)\right)\right|_{q}}{\left|H_{\phi_{k T} x, q}\left(d_{x} \phi_{k T}(\eta)\right)\right|_{q}} \\
& =\frac{\left|\left(A_{q}^{u}\right)^{k} H_{x, q}(\xi)\right|_{q}}{\left|\left(A_{q}^{u}\right)^{k} H_{x, q}(\eta)\right|_{q}}
\end{aligned}
$$

and then since $H_{x, q}(\eta) \in S(q)$ but $H_{x, q}(\xi) \notin S(q)$, the previous lemma tells us that

Now let $B$ denote the ball

$$
\lim _{k \rightarrow \infty} \frac{\left|d_{x} \phi_{k T}(\xi)\right|_{\phi_{k T} x}}{\left|d_{x} \phi_{k T}(\eta)\right|_{\phi_{k T} x}}=\infty .
$$

$$
B=\left\{x \in W^{s}(q): \operatorname{dist}(q, x) \leq 1\right\} .
$$

Using continuity and compactness of $B$, we see that the operator norm of $L^{u}$ with respect to the norm $|\cdot|$ on $E^{u} \mid B$ is uniformly bounded on $B$.

Suppose now for contradiction that there exists $x \in W^{s}(q)$ and $\xi \in S(x)$ with $L_{x}^{u} \xi \notin S(x)$. Then since $L_{x}^{u}$ is $\phi_{t}$-invariant we have

$$
L_{\phi_{k T} x}^{u}\left(d_{x} \phi_{k T}(\xi)\right)=d_{x} \phi_{k T}\left(L_{x}^{u} \xi\right)
$$

for all $k \geq 0$, and thus

$$
\lim _{k \rightarrow \infty} \frac{\left.\mid L_{\phi_{k T} x}^{u}\left(d_{x} \phi_{k T}(\xi)\right)\right)\left.\right|_{\phi_{k T} x}}{\left|d_{x} \phi_{k T}(\xi)\right|_{\phi_{k T} x}}=\infty .
$$


In other words, the operator norms with respect to $|\cdot|$ of the endomorphisms $L_{\phi_{k T} x}^{u}$ of $E^{u}\left(\phi_{k T} x\right)$ tends to infinity as $k \rightarrow \infty$, contradicting the fact that $\phi_{k T} x \in B$ for $k$ large enough.

Thus $L^{u}$ preserves $S$ over $E^{u} \mid W^{s}(q)$. To complete the proof we need to show $L^{u}$ preserves $S$ over all of $E^{u} \mid W^{+}(q)$. This however is clear, since $L^{u}$ is $\phi_{t}$-invariant.

Proposition 3.10. Given $x \in W^{+}(q)$ we have

$$
L_{x}^{u}=H_{q, x} \circ L_{q}^{u} \circ H_{x, q} \text { as maps } S(x) \rightarrow S(x) .
$$

In other words, when restricted to the subbundle $S$ over $W^{+}(q)$, the map $L^{u}$ 'commutes with holonomy'.

Proof. As before, since $L^{u}$ commutes with $d \phi_{t}$ it suffices to verify the assertion for $x \in W^{s}(q)$. Thus given $x \in W^{s}(q)$, define $C_{x} \in \operatorname{End} S$ by

$$
C_{x}=L_{x}^{u}-H_{q, x} \circ L_{q}^{u} \circ H_{x, q} .
$$

To complete the proof we show that $C_{x}=0$ for all $x \in W^{s}(q)$. To do this we shall show that the function $\beta$ defined on $W^{s}(q)$ by

$$
\beta(x)=\left\|C_{x}\right\|_{x}
$$

(where here $\|\cdot\|_{x}$ denotes the operator norm on $E^{u}(x)$ with respect to $\langle\cdot, \cdot\rangle_{x}$ ) is invariant under $\phi_{T}$. Since $\beta$ is continuous and $\beta(q)=0$ it then follows $\beta$ is identically zero, and hence $C_{x}=0$ for all $x \in W^{s}(q)$.

Since $L^{u}$ is $\phi_{t}$-invariant we have

$$
C_{\phi_{T} x}\left(d_{x} \phi_{T}(\xi)\right)=d_{x} \phi_{T}\left(C_{x}(\xi)\right)
$$

for all $x \in W^{s}(q)$ and $\xi \in S(x)$. Since (using (3.4))

$$
\begin{aligned}
\left|d_{x} \phi_{T}(\xi)\right|_{x} & =\left|H_{x, q}\left(d_{x} \phi_{T}(\xi)\right)\right|_{q} \\
& =\left|A_{q}^{u}\left(H_{x, q}^{+}(\xi)\right)\right|_{q} \\
& =\sigma\left|H_{x, q}^{+}(\xi)\right|_{q} \\
& =\sigma|\xi|_{x},
\end{aligned}
$$

for all $\xi \in S(x)$ and $x \in W^{s}(q)$, for $k \geq 0$ we have

$$
\begin{aligned}
\frac{\left|C_{\phi_{k T} x} \circ d_{x} \phi_{k T}(\xi)\right|_{\phi_{k T} x}}{\left|d_{x} \phi_{k T}(\xi)\right|_{\phi_{k T} x}} & =\frac{\left|d_{x} \phi_{k T} \circ C_{x}(\xi)\right|_{\phi_{k T} x}}{\sigma^{k}|\xi|_{x}} \\
& =\frac{\left|C_{x}(\xi)\right|_{x}}{|\xi|_{x}} ;
\end{aligned}
$$

which proves the assertions about the map $\beta$ stated above.

The point of all this work is the following result.

Corollary 3.11. Let $\rho_{0} \in \mathbb{C}$ be an eigenvalue of $\mathbb{L}_{q} \mid S_{\mathbb{C}}(q)$. Then the dimension of $P_{\rho_{0}}$ is strictly positive.

Proof. From the previous proposition it follows that the non-trivial subspace $Q_{\rho_{0}}(q) \cap$ $S(q)$ is contained in $P_{\rho_{0}}(q)$, and hence in the notation of Theorem 3.7, the integer $K=\operatorname{dim} P_{\rho_{0}}$ is strictly positive.

We conclude this section with the following construction, again due to Hamenstädt [19, p686]. If $V$ is a real vector space and $V_{i} \subseteq V$ are even dimensional subspaces admitting almost complex structures $\mathbb{J}_{i}$, let $\bar{\bigcap}_{i}\left(V_{i}, \mathbb{J}_{i}\right)$ denote the largest subspace $W \subseteq \bigcap_{i} V_{i}$ which is invariant under the $\mathbb{J}_{i}$ and such that $\mathbb{J}_{i}\left|W=\mathbb{J}_{j}\right| W$ for all $i, j$. We call $\bar{\bigcap}_{i}\left(V_{i}, \mathbb{J}_{i}\right)$ the complex intersection of the $\left(V_{i}, \mathbb{J}_{i}\right)$. 
Now suppose the $\rho_{0}$ we found above happens to lie in $\mathbb{C} \backslash \mathbb{R}$. Then if we consider the operator

$$
\mathbb{J}:=\frac{1}{\operatorname{Im} \rho_{0}}\left(L^{u}-\left(\operatorname{Re} \rho_{0}\right) \operatorname{Id}\right),
$$

it is easy to see that $\mathbb{J}$ defines an almost complex structure on $Q_{\rho_{0}}$.

If follows that if we define a new bundle $P_{\rho_{0}}^{\mathbb{C}}$ over all of $\Sigma$ by setting

$$
P_{\rho_{0}}^{\mathbb{C}}(x):=\bar{\bigcap}\left\{\left(H_{y, x} Q_{\rho_{0}}(y), \mathbb{J}_{y}\right): y \in W^{s}(x)\right\},
$$

then the same proof as above shows that $P_{\rho_{0}}^{\mathbb{C}}$ is a continuous $\phi_{t}$-invariant subundle of $E^{u}$ over $\Sigma$ of positive dimension. We shall use this observation later.

\section{North-South Dynamics}

In this section we return to the situation described in the introduction. The main goal of this section is to prove Theorem 4.2 below; we will use heavily the assumption that $M$ admits a metric of negative curvature. As stated in the introduction however it should be possible to prove Theorem 4.2 without using this assumption.

Throughout this section $(M, g)$ denotes a closed $n$-dimensional Riemannian manifold with tangent bundle $\pi: T M \rightarrow M$. We first begin with a quick summary of the geometry of the tangent bundle that we will need throughout what follows.

The geometry of $T M$. The vertical bundle $V \subseteq T T M$ is given by

$$
V(v)=\operatorname{ker}\left\{d_{v} \pi: T_{v} T M \rightarrow T_{x} M\right\},
$$

where for convenience throughout this paragraph an arbitrary vector $v \in T M$ is assumed to lie in $T_{x} M$. The Riemannian metric $g$ on $M$ determines a direct summand $H$ of the vertical bundle $V$, called the horizontal bundle together with isomorphisms

$$
T_{v} T M \cong H(v) \oplus V(v) \cong T_{x} M \oplus T_{x} M .
$$

Let $\nabla$ denote the Levi-Civita connection on $(M, g)$. We make this isomorphism explicit as follows: given $\xi \in T_{v} T M$ associate a point $\left(\xi_{H}, \xi_{V}\right) \in T_{x} M \oplus T_{x} M$, where

and

$$
\xi_{H}=d_{v} \pi(\xi)
$$

$$
\xi_{V}=K_{v}(\xi) .
$$

Here $K: T T M \rightarrow T M$ is the connection map of $\nabla$, defined as follows: given $\xi \in T_{v} T M$, choose a curve $Z:(-\varepsilon, \varepsilon) \rightarrow T M$ such that $Z(0)=v$ and $\dot{Z}(0)=\xi$. Then

$$
K_{v}(\xi)=\nabla_{t} Z(0),
$$

where $\nabla_{t}$ denotes the covariant derivative along the curve $\pi \circ Z$.

$H(v)$ is thus defined to be the set of $\xi \in T_{v} T M$ such that $\xi_{V}=0$, and similarly $V(v)$ is simply the set of $\xi \in T_{v} T M$ such that $\xi_{H}=0$. Clearly the map $\xi \mapsto \xi_{H}$ defines an isomorphism $H(v) \rightarrow T_{x} M$ and similarly $\xi \mapsto \xi_{V}$ defines an isomorphism $V(v) \rightarrow T_{x} M$. In general we shall slightly abuse notation and write $\xi=\left(\xi_{H}, \xi_{V}\right)$ to indicate this identification.

It is easy to see that given $\xi, \eta \in T T M$ we have

$$
\omega_{0}(\xi, \eta)=\left\langle\xi_{H}, \eta_{V}\right\rangle-\left\langle\xi_{V}, \eta_{H}\right\rangle
$$

where as before $\omega_{0}$ denotes the canonical symplectic form on $T M$. We define the Sasaki metric $g_{T M}$ on $T M$ by setting

$$
\langle\xi, \eta\rangle_{T M}:=\left\langle\xi_{H}, \eta_{H}\right\rangle+\left\langle\xi_{V}, \eta_{V}\right\rangle,
$$


so that $\omega_{0}$ and $g_{T M}$ are compatible.

North-South dynamics. As before, let $H: T M \rightarrow M$ denote the energy Hamiltonian $(x, v) \mapsto \frac{1}{2}|v|^{2}$, and let $F_{1}$ denote the symplectic gradient of $H$ with respect to $\omega_{1}$. Let $\phi_{t}^{1}: T M \rightarrow T M$ denote the flow of $F_{1}$. Let $\Sigma_{k}=H^{-1}(k)$ denote a closed energy level set, where $k$ is a regular value of $H$, and assume that $\phi_{t}^{1} \mid \Sigma_{k}$ is Anosov (we do not need to assume $C^{1}$ weak (un)stable bundles at this point). Write the Anosov splitting of $T \Sigma_{k}$ as

$$
T \Sigma_{k}=\mathbb{R} F_{1} \oplus E_{1}^{s} \oplus E_{1}^{u} .
$$

We first quote the following theorem from [30, which will very useful in what follows.

Theorem 4.1. If $\Sigma_{k}$ is an Anosov energy level then the weak (un)stable bundles $E_{1}^{+}$and $E_{1}^{-}$are transverse to the vertical subbundle $V$.

Now let $\widetilde{M}$ denote the universal covering of $M$, and let $\widetilde{\Sigma}_{k}$ denote the pullback of $\Sigma_{k}$ to $T \widetilde{M}$. Then $\widetilde{\Sigma}_{k}$ is a smooth connected hypersurface of $T \widetilde{M}$ that intersects each tangent space $T_{x} \widetilde{M}$ in a sphere containing the origin in its interior (since the same is true of $\Sigma_{k}$ ). Let $\widetilde{\sigma}$ denote the pullback of $\sigma$ to $\widetilde{M}$ and let $\widetilde{\omega}_{0}$ denote the natural symplectic form on $T \widetilde{M}$. Let $\widetilde{\omega}_{1}:=\widetilde{\omega}_{0}+\widetilde{\pi}^{*} \widetilde{\sigma}$, where $\widetilde{\pi}: T \widetilde{M} \rightarrow \widetilde{M}$ is the footpoint map. We will let $\widetilde{F}_{1}$ denote the symplectic gradient of the lifted Hamiltonian $(x, v) \mapsto \frac{1}{2}|v|^{2}$ with respect to $\widetilde{\omega}_{1}$. We will write $\widetilde{\phi}_{t}^{1}: T \widetilde{M} \rightarrow T \widetilde{M}$ for the flow of $\widetilde{F}_{1}$. By assumption $\widetilde{\phi}_{t}^{1} \mid \widetilde{\Sigma}_{k}$ is Anosov, and we will write the Anosov splitting as

$$
T \widetilde{\Sigma}_{k}=\mathbb{R} \widetilde{F}_{1} \oplus \widetilde{E}_{1}^{s} \oplus \widetilde{E}_{1}^{u} .
$$

Similarly let us denote by $\widetilde{V}$ and $\widetilde{H}$ denote the vertical and horizontal subbundles of $T T \widetilde{M}$.

As before let $\mathcal{W}^{s}, \mathcal{W}^{u}, \mathcal{W}^{+}$and $\mathcal{W}^{-}$denote the four foliations of $\Sigma_{k}$ defined by the subbundles $E^{s}, E^{u}, E^{+}$and $E^{-}$respectively. We can lift these to foliations $\widetilde{\mathcal{W}}^{s}, \widetilde{\mathcal{W}}^{u}, \widetilde{\mathcal{W}}^{+}$and $\widetilde{\mathcal{W}}^{-}$of $\widetilde{\Sigma}_{k}$. Let $\mathcal{L}^{+}=\widetilde{\Sigma}_{k} / \widetilde{\mathcal{W}}^{+}$and $\mathcal{L}^{-}=\widetilde{\Sigma}_{k} / \widetilde{\mathcal{W}}^{-}$denote the spaces of weak stable and unstable leaves respectively. The fundamental group $\pi_{1}(M)$ (regarded as covering transformations of $\widetilde{M}$ ) acts on $\widetilde{\Sigma}_{k}$ freely and properly discontinuously by permuting the orbits of $\widetilde{\phi}_{t}^{1}$. Since elements of $\pi_{1}(M)$ act by isometries, the action on $\widetilde{\Sigma}_{k}$ must send weak (un)stable leaves to weak (un)stable leaves, and thus $\pi_{1}(M)$ induces an action on $\mathcal{L}^{+}$and $\mathcal{L}^{-}$.

The aim of this section is to prove the following result.

Theorem 4.2. Suppose $M$ admits a metric of negative curvature. The fundamental group $\pi_{1}(M)$ acts on both $\mathcal{L}^{+}$and $\mathcal{L}^{-}$as a 'North-South dynamic'. By this we mean the following: for all $\varphi \in \pi_{1}(M)$, there exists two fixed leaves $\widetilde{W}_{1}^{+}, \widetilde{W}_{2}^{+} \in \mathcal{L}^{+}$and two fixed leaves $\widetilde{W}_{1}^{-}, \widetilde{W}_{2}^{-} \in \mathcal{L}^{-}$such that for all $\widetilde{W}^{ \pm} \in \mathcal{L}^{ \pm}$it holds that

$$
\lim _{n \rightarrow \infty} \varphi^{n}\left[\widetilde{W}^{ \pm}\right]=\widetilde{W}_{1}^{ \pm}, \quad \lim _{n \rightarrow \infty} \varphi^{-n}\left[\widetilde{W}^{ \pm}\right]=\widetilde{W}_{2}^{ \pm}
$$

Consider the fibration

$$
\pi \mid \Sigma_{k}: \Sigma_{k} \rightarrow M
$$

of $(n-1)$-spheres. As a direct consequence of Theorem 4.1. we see that the foliations $\mathcal{W}^{+}$and $\mathcal{W}^{-}$are transverse to the fibres of the fibration $\Sigma_{k} \rightarrow M$. This implies that $\widetilde{\mathcal{W}}^{+}$and $\widetilde{\mathcal{W}}^{-}$are transverse to the fibration $\widetilde{\pi} \mid \widetilde{\Sigma}_{k}: \widetilde{\Sigma}_{k} \rightarrow \widetilde{M}$.

Given $x \in \widetilde{M}$, the fibre $\left(\widetilde{\pi} \mid \widetilde{\Sigma}_{k}\right)^{-1}(x)$ is compact. Thus we can apply the following theorem of Ehresmann. 
Theorem 4.3. Let $F \rightarrow E \stackrel{p}{\rightarrow} B$ be a fibre bundle and $\mathcal{F}$ a foliation of $E$ transverse to the fibres. Suppose $F$ is compact. Then for every leaf $L$ of $\mathcal{F}, p \mid L: L \rightarrow B$ is a covering map.

For a proof, see [6, p91]. Now fix $v \in \widetilde{\Sigma}_{k}$. Since $\widetilde{M}$ is simply connected, $\widetilde{\pi} \mid \widetilde{W}^{+}(v)$ is a diffeomorphism, and thus $\widetilde{W}^{+}(v)$ simply connected. Thus $\widetilde{W}^{+}(v)$ intersects each fibre of the fibration $\widetilde{\Sigma}_{k} \rightarrow \widetilde{M}$ in precisely one point, and thus each leaf $\widetilde{W}^{+}(v)$ is diffeomorphic to $\widetilde{M}$. Thus $\widetilde{M}$ is diffeomorphic to $\mathbb{R}^{n}$, and the space of stable leaves $\mathcal{L}^{+}$can be identified topologically with the $(n-1)$-sphere. Of course the same applies to $\mathcal{L}^{-}$.

Now we recall the concept of the ideal boundary of $\widetilde{M}$. For this, let $g_{0}^{\prime}$ denote a metric of negative curvature on $M$ (whose existence we assume in this section), and lift $g_{0}^{\prime}$ to a metric $g_{0}$ on $\widetilde{M}$ of negative curvature.

Definition 4.4. The ideal boundary $\widetilde{M}_{g_{0}}(\infty)$ of $\left(\widetilde{M}, g_{0}\right)$ is given by $\widetilde{M}_{g_{0}}(\infty):=$ $\Lambda_{g_{0}}(\widetilde{M}) / \sim$, where $\Lambda_{g_{0}}(\widetilde{M})$ denotes the set of $g_{0}$-geodesics 1$]$ $c_{1} \sim c_{2}$ if and only if

$$
\operatorname{dist}_{\mathrm{HD}}\left(c_{1}\left[\mathbb{R}^{+}\right], c_{2}\left[\mathbb{R}^{+}\right]\right)<\infty
$$

here dist $_{\mathrm{HD}}$ denotes the Hausdorff distance defined by

$$
\operatorname{dist}_{\mathrm{HD}}(U, V):=\inf \{r \in \mathbb{R}: U \subseteq B(V, r), V \subseteq B(U, r)\},
$$

where $U, V \subseteq \widetilde{M}$ and $B(U, r):=\left\{x \in \widetilde{M}: \operatorname{dist}_{g_{0}}(x, U) \leq r\right\}$.

Given $x \in \widetilde{M}$ and $v \in T_{x} \widetilde{M}$, let $c_{v}: \mathbb{R} \rightarrow \widetilde{M}$ denotes the unique $g_{0}$-geodesic adapted to $v$, and let $c_{v}(\infty) \in \widetilde{M}_{g_{0}}(\infty)$ denote the corresponding element of $\widetilde{M}_{g_{0}}(\infty)$. If $c_{v}^{-1}$ is the geodesic obtained by going along $c_{v}$ backwards, let $c_{v}(-\infty)$ denote the element of $\widetilde{M}_{g_{0}}(\infty)$ corresponding to $c_{v}^{-1}$.

Let $S^{g_{0}} \widetilde{M}$ denote the unit sphere bundle of $\left(\widetilde{M}, g_{0}\right)$. Fix a point $x \in \widetilde{M}$, and consider the map $s_{x}: S_{x}^{g_{0}} \widetilde{M} \rightarrow \widetilde{M}_{g_{0}}^{g_{0}}(\infty)$ sending $v \mapsto c_{v}(\infty)$. Then $s_{x}$ is a bijection, and we define a topology on $\widetilde{M}_{g_{0}}(\infty)$ so that that $s_{x}$ becomes a homeomorphism; thus $\widetilde{M}_{g_{0}}(\infty) \cong S^{n-1}$. This topology is independent of the choice of $x$, since $s_{y} \circ s_{x}^{-1}: S_{x}^{g_{0}} \widetilde{M} \rightarrow S_{y}^{g_{0}} \widetilde{M}$ is a homeomorphism.

The next thing we require is the concept of a quasi-geodesic.

Definition 4.5. A curve $\gamma:[a, b] \rightarrow \widetilde{M}$ is an quasi-geodesic of $\left(\widetilde{M}, g_{0}\right)$ if there exist $P, Q \in \mathbb{R}^{+}$such that

$$
\frac{1}{P}|s-t|-Q \leq \operatorname{dist}_{g_{0}}(\gamma(s), \gamma(t)) \leq P|s-t|+Q
$$

for all $s, t \in[a, b]$. If we need to be explicit about the constants $P, Q$, we call such a quasi-geodesic a $(P, Q)$-quasi-geodesic.

We now quote two theorems which explain why this is relevant to the situation in hand. The first is due to Peyerimhoff and Siburg ([31, Theorem 2.9]).

Theorem 4.6. Suppose $\phi_{t}^{1} \mid \Sigma_{k}$ is Anosov. Then there exists a constant $P_{k} \in \mathbb{R}^{+}$ such that the projection to $\widetilde{M}$ of any orbit of $\widetilde{\phi}_{t}^{1}$ is a $\left(P_{k}, 0\right)$-quasi-geodesic.

\footnotetext{
${ }^{1}$ For clarity we will use the letter $c$ to stand for $g_{0}$-geodesics and $\gamma$ for the projection to $\widetilde{M}$ of flow lines of $\widetilde{\phi}_{t}^{1}$.
} 
Remark 4.7. In fact, Theorem 4.6 is stated in a somewhat different form in [31: there they assert that when $k$ is greater than a certain critical value $c(g, \sigma)$ known as Mañés critical value then the projection to $\widetilde{M}$ of any minimizing orbit of $\widetilde{\phi}_{t}^{1}$ is a quasi-geodesic. See for instance [9, 5] for the definition of $c(g, \sigma)$, where it is proved that when $\phi_{t}^{1}$ is Anosov, it necessarily holds that $k>c(g, \sigma)$, and that in this case, every orbit of $\widetilde{\phi}_{t}^{1}$ is minimizing.

We can build the quasi-ideal boundary $\widetilde{M}_{g_{0}}^{*}(\infty)$ in much the same way using quasi-geodesics. If $\gamma: \mathbb{R} \rightarrow \widetilde{M}$ is an $g_{0}$-quasi-geodesic, we write $\gamma^{*}(\infty)$ to denote the corresponding element of $\widetilde{M}_{g_{0}}^{*}(\infty)$. Note that any geodesic is automatically a quasi-geodesic, and thus we have a natural map $\widetilde{M}_{g_{0}}(\infty) \hookrightarrow \widetilde{M}_{g_{0}}^{*}(\infty)$ carrying an equivalence class of geodesics to the corresponding equivalence class of quasigeodesics. This is the second theorem we quote here; a proof may be found in [27. Theorem 2.2].

Theorem 4.8. The inclusion $\widetilde{M}_{g_{0}}(\infty) \hookrightarrow \widetilde{M}_{g_{0}}^{*}(\infty)$ is a bijection.

We will use this to show the following key result, whose proof is essentially that of Theorem 2.12 in [27. The result however is originally due to Gromov (see [17]), and also independently due to Ghys ([16, Theorem 4.5]). Let $\psi_{t}$ denote the geodesic flow of $\left(\widetilde{M}, g_{0}\right)$ and let $\psi_{t}^{\prime}$ denote the geodesic flow of $\left(M, g_{0}^{\prime}\right)$. Let $S^{g_{0}^{\prime}} M$ denote the unit sphere bundle of $\left(M, g_{0}^{\prime}\right)$.

Theorem 4.9. $\phi_{t}^{1} \mid \Sigma_{k}$ and $\psi_{t}^{\prime} \mid S^{g_{0}^{\prime}} M$ are topologically conjugate.

Proof. Given $v \in \widetilde{\Sigma}_{k}$, let $\gamma_{v}:=\widetilde{\pi} \circ \widetilde{\phi}_{t}^{1} v$. Then $\gamma_{v}$ determines an element $\gamma_{v}^{*}(\infty) \in$ $\widetilde{M}_{g_{0}}^{*}(\infty)$ by Theorem 4.6, and thus by Theorem 4.8 a unique element $\xi_{v} \in \widetilde{M}_{g_{0}}(\infty)$. Let $\xi_{v}^{-1} \in \widetilde{M}_{g_{0}}(\infty)$ denote the element corresponding to $\gamma_{v}^{*}(-\infty) \in \widetilde{M}_{g_{0}}^{*}(\infty)$.

Suppose $\zeta, \xi \in \widetilde{M}_{g_{0}}(\infty)$. Then there exists a unique $g_{0}$-geodesic $c$ such that $c(\infty)=\zeta$ and $c(-\infty)=\xi$. Let $\mathbb{P}(c): \widetilde{M} \rightarrow \widetilde{M}$ denote orthogonal projection onto $c$, and use this to define a map $\mathbb{P}(\zeta, \xi): \widetilde{M} \rightarrow S^{g_{0}} \widetilde{M}$ by

$$
\mathbb{P}(\zeta, \xi)(x)=\dot{c}(t) \quad \text { where } \quad \mathbb{P}(c)(x)=c(t) .
$$

Now define $G_{0}: \widetilde{\Sigma}_{k} \rightarrow S^{g_{0}} \widetilde{M}$ by setting

$$
G_{0}(v):=\mathbb{P}\left(\xi_{v}, \xi_{v}^{-1}\right)(\tilde{\pi} v),
$$

Then $G_{0}$ is continuous and surjective but in general not injective: there may exist two points $v, v^{\prime}$ on the same orbit of $\widetilde{\phi}_{t}^{1}$ that have the same orthogonal projection onto the $g_{0}$-geodesic $c$ determined by $\xi_{v}=\xi_{v^{\prime}}$ and $\xi_{v}^{-1}=\xi_{v^{\prime}}^{-1}$. In order to achieve injectivity we 'average' $G_{0}$. For this look at the map $\rho: \mathbb{R} \times \widetilde{\Sigma}_{k} \rightarrow S^{g_{0}} \widetilde{M}$ defined by

$$
G_{0}\left(\widetilde{\phi}_{t}^{1} v\right)=\psi_{\rho(t, v)}\left(G_{0}(v)\right)
$$

Then $\rho$ satisfies the cocycle property, that is,

$$
\rho\left(t+t^{\prime}, v\right)=\rho\left(t, \widetilde{\phi}_{t^{\prime}}^{1} v\right)+\rho\left(t^{\prime}, v\right)
$$

as is easily checked. Now choose $\tau \in \mathbb{R}^{+}$such that $\rho(\tau, v)>0$ for all $v \in \widetilde{\Sigma}_{k}$, and then let $r(v)$ denote the average

$$
r(v):=\frac{1}{\tau} \int_{0}^{\tau} \rho(t, v) d t
$$

Next define $G_{\tau}: \widetilde{\Sigma}_{k} \rightarrow S^{g_{0}} \widetilde{M}$ by

$$
G_{\tau}(v)=\psi_{r(v)}\left(G_{0}(v)\right)
$$


We claim that $G_{\tau}$ is injective. For this observe that if

$$
f(t):=r\left(\widetilde{\phi}_{t}^{1} v\right)+\rho(t, v)
$$

then $f$ is monotone increasing. Indeed,

$$
\begin{aligned}
f^{\prime}(t) & =\frac{1}{\tau} \int_{0}^{\tau} \rho^{\prime}(u+t, v) d u \\
& =\frac{1}{\tau}(\rho(\tau+t, v)-\rho(t, v)) \\
& =\frac{1}{\tau} \rho\left(\tau, \widetilde{\phi}_{t}^{1} v\right)>0 .
\end{aligned}
$$

The claim then follows from the computation

$$
\begin{aligned}
G_{\tau}\left(\widetilde{\phi}_{t}^{1} v\right) & =\psi_{r\left(\psi_{t} v\right)}\left(G_{0}\left(\widetilde{\phi}_{t}^{1} v\right)\right) \\
& =\psi_{r\left(\psi_{t} v\right)+\rho(t, v)}\left(G_{0}(v)\right) \\
& =\psi_{f(t)}\left(G_{0}(v)\right)
\end{aligned}
$$

Finally, in order to deduce the stronger statement that $\phi_{t}^{1} \mid \Sigma_{k}$ and $\psi_{t}^{\prime} \mid S^{g_{0}^{\prime}} M$ are also topologically conjugate, one simply notes that $G_{0}$ is obviously equivariant under the action of $\pi_{1}(M)$, and hence so is $G_{\tau}$; thus $G_{\tau}$ descends to $M$ to define an orbit equivalence $G_{\tau}^{\prime}$ from $\phi_{t}^{1} \mid \Sigma_{k}$ and $\psi_{t}^{\prime} \mid S^{g_{0}^{\prime}} M$.

It is now easy to prove Theorem 4.2. Indeed, it is well known (see for instance [26. Theorem 3.8.13]) that Theorem 4.2 holds in the case of a geodesic flow of a negatively curved manifold. Thus if $M$ admits a metric $g_{0}^{\prime}$ of negative curvature, Theorem 4.9 gives us an orbit equivalence between $\phi_{t}^{1} \mid \Sigma_{k}$ and the geodesic flow $\psi_{t}^{\prime}$ of $\left(M, g_{0}^{\prime}\right)$, and via this orbit equivalence we see Theorem 4.2 holds in our case too.

\section{Proof of Theorem A}

We will now prove Theorem A. Our proof of the theorem will depend on the parity of $n$; moreover, a separate argument will be required to deal with the cases $n=3$ and $n=7$. We will start with the case where $n$ is even.

The case when $n$ is an even integer. In the even dimensional case, recall that we assume $\phi_{t}^{1} \mid \Sigma_{k}$ is $1 / 2$-pinched. Since $\left(\Sigma_{k}, \omega_{1}\right)$ is stable, there exists an invariant subbundle $P_{\rho_{0}}$ of $E^{u}$ as constructed in Section 3 , see (3.1). The maximal integral submanifolds of $P_{\rho_{0}}$ define a foliation $\mathcal{P}(v)$ of class $C^{1}$ on $W^{u}(v)$. Since $P$ is invariant under parallel transport, it is also invariant under holonomy transport and thus the foliations $\mathcal{P}(v)$ glue together to give a foliation $\mathcal{P}$ of class $C^{1}$ on $\Sigma_{k}$ that is invariant under the parallel transport of $\nabla$ and thus also the holonomy maps. Thus $\mathcal{P}$ can be lifted to $\widetilde{\Sigma}_{k}$ and then projected to a foliation $\mathcal{P}^{\prime}$ of $\mathcal{L}^{+}$of positive dimension.

Since $\mathcal{P}$ is invariant under $\phi_{t}, \mathcal{P}^{\prime}$ is invariant under the action of $\pi_{1}(M)$. Here Theorem 4.2 of the previous section comes into play: $\pi_{1}(M)$ acts on $\mathcal{L}^{+}$as a NorthSouth dynamics. A theorem of Foulon [15] states that there are no non-trivial $C^{0}$ foliations of the sphere $S^{n-1}$ which are invariant under North-South dynamics. Since we know that $\mathcal{P}^{\prime}$ is of positive dimension, we must have $\mathcal{P}^{\prime}=\mathcal{L}^{+}$, and hence the subbundle $P_{\rho_{0}}$ is equal to $E^{u}$. From this it is easy to deduce that $d \lambda=\rho_{0} \omega_{1}$, where $\rho_{0} \in \mathbb{R}$ defines $P_{\rho_{0}}$; see (3.1).

To complete the proof in this case it remains to rule out the possibility that $\rho_{0}=0$. Suppose for contradiction that this is the case. Then the 1 -form $\lambda$ is 
closed. Let $\mu$ denote a Borel probability measure on $\Sigma_{k}$. Recall that $\mu$ determines a 1 -current $l_{\mu}$ by

$$
l_{\mu}(\beta)=\int_{\Sigma_{k}} \beta\left(F_{1}\right) d \mu: \beta \in \Omega^{1}\left(\Sigma_{k}\right) .
$$

We say that $\mu$ is exact as a current if $l_{\mu}(\beta)=0$ whenever $\beta$ is closed. Now let $\mu_{L}$ denote the Liouville measure of $\Sigma_{k}$ (defined precisely below). Then by Lemma 5.1 below, $\mu_{L}$ is exact as a current. But

$$
l_{\mu_{L}}(\lambda)=\int_{\Sigma_{k}} \lambda\left(F_{1}\right) d \mu_{L} \neq 0 ;
$$

contradiction. Thus $\rho_{0} \neq 0$; this completes the proof in the even dimensional case.

For completeness let us give a precise definition of the Liouville measure $\mu_{L}$ and prove that it is indeed exact as a current. Whilst in general there may exist many invariant volume forms on an energy level $\Sigma_{k}$, and thus many invariant probability measures, in the special case where the energy level $\Sigma_{k}$ is Anosov, the Liouville measure is the unique smooth invariant probability measure. It can be defined as follows. Let $X \in T T M \mid \Sigma_{k}$ denote a vector field such that $\omega_{1}\left(X, F_{1}\right)=1$ (such a vector field always exists since $\Sigma_{k}$ is a regular energy level). Observe that

$$
i_{F_{1}} i_{X} \omega_{1}^{n} \mid \Sigma_{k}=\omega_{1}^{n-1}
$$

and hence if $\Theta:=i_{X} \omega_{1}^{n}$ then $\Theta$ is a volume form on $\Sigma_{k}$. Now observe that $\omega_{1}^{n-1}$ is exact for $n \geq 3$. Indeed

$$
\omega_{1}^{n-1}=\left(\omega_{0}+\pi^{*} \sigma\right)^{n-1}=\left(\omega_{0}\right)^{n-1}+(n-1) \pi^{*} \sigma \wedge\left(\omega_{0}\right)^{n-2} .
$$

On the right-hand side the first term is exact. For $n \geq 3$ the second term is exact as well and the claim follows (when $n=2, \omega_{1}$ is exact if $M$ is not the 2-torus, but we do not need this here). Then $i_{F_{1}} \Theta=d \tau$ and $\phi_{t}^{1}$ preserves the volume form $\Theta$. Let $\mu_{L}$ denote the smooth invariant probability measure induced by $\Theta ; \mu_{L}$ is called the Liouville measure.

Lemma 5.1. The Liouville measure $\mu_{L}$ of $\Sigma_{k}$ is exact as a current.

Proof. Let $\beta \in \Omega^{1}\left(\Sigma_{k}, \mathbb{R}\right)$ denote any closed 1 -form and let $A$ be the integral of $\Theta$. Then

$$
\begin{aligned}
A l_{\mu_{L}}(\beta) & =A \int_{\Sigma_{k}} \beta\left(F_{1}\right) d \mu_{L} \\
& =\int_{\Sigma_{k}} \beta\left(F_{1}\right) \Theta \\
& =\int_{\Sigma_{k}} i_{F_{1}} \Theta \wedge \beta \\
& =\int_{\Sigma_{k}} d \tau \wedge \beta \\
& =\int_{\Sigma_{k}} d(\tau \wedge \beta),
\end{aligned}
$$

and this last integral is zero by Stokes' theorem.

The case when $n$ is an odd integer. We now proceed to the second case, where $n$ is odd. We no longer need to assume that $\phi_{t}^{1} \mid \Sigma_{k}$ is $1 / 2$-pinched, only that the weak (un)stable bundles are of class $C^{1}$. The next result is from [13, Lemma 2].

Lemma 5.2. Let $K \subseteq \widetilde{E}_{1}^{u}$ be a continuous distribution of $k$-dimensional planes defined everywhere on $\widetilde{\Sigma}_{k}$. Then $K$ projects onto a continuous field $Q$ of $k$-planes on $S^{n-1}$. 
Proof. Let $C \subseteq \widetilde{M}$ denote a closed submanifold of $\widetilde{M}$ diffeomorphic to $S^{n-1}$. For each $x \in C$, let $\nu(x) \in T_{x} \widetilde{M}$ denote the inward pointing normal to $C$ at $x$. Thus $K(\nu(x)) \subseteq \widetilde{E}_{1}^{u}(\nu(x))$. Then note that given $x \in C$ and $v \in \widetilde{E}_{1}^{s}(x)$, the map $d_{v} \widetilde{\pi} \mid \widetilde{E}_{1}^{u}: \bar{E}_{1}^{u}(v) \rightarrow v^{\perp} \subseteq T_{x} \widetilde{M}$ is a linear isomorphism. The desired continuous field $Q$ of $k$-planes is then given by

$$
Q(x):=d_{\nu(x)} \tilde{\pi}[K(\nu(x))] \subseteq T_{x} C .
$$

For the case where $M$ is odd dimensional we will use the bundle $P_{\rho_{0}}$ defined by (3.5), where this time $\rho_{0} \in \mathbb{C}$ is given by Corollary 3.11. In this case however the argument is initially simpler. Indeed, by the following topological result, after lifting $P_{\rho_{0}}$ to a continuous distribution on $\widetilde{E}_{1}^{u}$, we see immediately that $P_{\rho_{0}}=E_{1}^{u}$.

Theorem 5.3. For $n$ odd, $S^{n-1}$ admits no $k$-plane distribution for $1 \leq k \leq n-2$.

See for instance [32, Theorem 27.18] for a proof.

Remark 5.4. We could alternatively deduce the same result by observing as before that the space of leaves $\mathcal{L}^{+}:=\widetilde{\Sigma}_{k} / \widetilde{\mathcal{W}}^{+}$is topologically an $(n-1)$-sphere, and since $P_{\rho_{0}}$ is invariant under the holonomy transport, $P_{\rho_{0}}$ determines a continuous distribution of $k$-planes on $S^{n-1}$. The proof given above does not use the fact that $P_{\rho_{0}}$ is invariant under holonomy transport.

Unfortunately this does not quite nail the result as in the even dimensional case. If $\rho_{0} \in \mathbb{R}$, we deduce $L^{u}=\rho_{0} \mathrm{Id}$, and the desired contradiction follows just as in the even-dimensional case.

If however $\rho_{0} \notin \mathbb{R}$ then more work is required. Consider the $\phi_{t}^{1}$-invariant almost complex structure $\mathbb{J}$ on $E^{u}$ of class $C^{1}$ defined by

$$
\mathbb{J}:=\frac{1}{\operatorname{Im} \rho_{0}}\left(L^{u}-\left(\operatorname{Re} \rho_{0}\right) \operatorname{Id}\right) .
$$

Lifting $\mathbb{J}$ to an almost complex structure on $\widetilde{E}_{1}^{u}$, and then using the construction from Lemma 5.2, we see that $\mathbb{J}$ induces an almost complex structure on $S^{n-1}$. This immediately implies that $n=3$ or $n=7$, since the only spheres that admit almost complex structures are $S^{2}$ and $S^{6}$.

It thus remains to eliminate the cases $n=3$ and $n=7$, and we will tackle these separately.

The case where $n=3$ or $n=7$. The first step in the proof of these two special cases is to show that the existence of a $\phi_{t}^{1}$-invariant almost complex structure $\mathbb{J}$ on $E^{u}$ forces both $E^{s}$ and $E^{u}$ to be of class $C^{\infty}$. The next two results are due to Hamenstädt; see Corollary 2.11 and Corollary 2.12 of [19].

Lemma 5.5. The almost complex structure $\mathbb{J}$ on $E^{u}$ is parallel with respect to the Kanai connection.

Proof. Since $\mathbb{J}$ is $\phi_{t}^{1}$-invariant and $\nabla_{F_{1}}=L_{F_{1}}$, we certainly have $\nabla_{F_{1}} \mathbb{J}=0$ and thus it suffices to show that $\nabla_{X_{s}} \mathbb{J}=0$ for all $X_{s} \in \Gamma\left(E^{s}\right)$ and $\nabla_{X_{u}} \mathbb{J}=0$ for all $X_{u} \in \Gamma\left(E^{u}\right)$. We know that $\mathbb{J}$ is invariant under the holonomy maps $H_{x, y}$ (since otherwise the subbundle $P_{\rho_{0}}^{\mathbb{C}}$ from (3.5) would be of positive dimension this contradict the fact that an even dimensional sphere does not have non-trivial subbundles) and thus as holonomy transport is the same as parallel transport for $\nabla$, we see that $\nabla_{X_{s}} \mathbb{J}=0$ for all $X_{s} \in \Gamma\left(E^{s}\right)$. We can define a new almost complex structure $\mathbb{J}^{\prime}$ on $E^{s}$ by the equation

$$
\omega\left(X_{u}, \mathbb{J}^{\prime} X_{s}\right)=\omega\left(\mathbb{J} X_{u}, X_{s}\right) .
$$


Then if $Y_{s} \in \Gamma\left(E^{s}\right)$, applying $Y_{s}$ to the previous equation and using that $\nabla \omega=0$ shows that

$$
\omega\left(X_{u},\left(\nabla_{Y_{s}} \mathbb{J}^{\prime}\right) X_{s}\right)=\omega\left(\left(\nabla_{Y_{s}} \mathbb{J}\right) X_{u}, X_{s}\right),
$$

and thus $\nabla_{Y_{s}} \mathbb{J}^{\prime}=0$.

But now the point is the following: we could repeat all of what we have done above but working with $L^{s}$ not $L^{u}$, and thus obtain an almost complex structure $\widetilde{\mathbb{J}}$ on $E^{s}$. The same argument would then show that this almost complex structure $\widetilde{\mathbb{J}}$ is parallel on $E^{u}$, that is, $\nabla_{X_{u}} \widetilde{\mathbb{J}}=0$ for $X_{u} \in \Gamma\left(E^{u}\right)$. But straight from the definition, it is clear that $\widetilde{\mathbb{J}}=\mathbb{J}^{\prime}$, and thus $\mathbb{J}^{\prime}$ is parallel on $E^{u}$ as well. But then taking $Y_{u} \in \Gamma\left(E^{u}\right)$ and plugging it into (5.2), we see that $\nabla_{Y_{u}} \mathbb{J}=0$, and this completes the proof.

Theorem 5.6. Suppose the strong unstable bundle $E_{1}^{u}$ admits an almost complex structure $\mathbb{J}$ defined as in (5.1). Then $\Sigma_{k}$ admits a real analytic structure, for which the strong (un)stable bundles $E_{1}^{s}$ and $E_{1}^{u}$ are both real analytic. Moreover, this real analytic structure is $C^{1}$ diffeomorphic to the underlying smooth structure of $\Sigma_{k}$.

Before starting the proof, let us recall the following facts about the spaces of leaves. Recall that the space $\mathcal{L}^{+}$and $\mathcal{L}^{-}$of leaves are defined to be the quotient spaces $\mathcal{L}^{+}:=\widetilde{\Sigma}_{k} / \sim_{+}$and $\mathcal{L}^{-}:=\widetilde{\Sigma}_{k} / \sim_{-}$, where $\sim_{ \pm}$are the equivalence relations on $\widetilde{\Sigma}_{k}$ defined by $v \sim_{ \pm} w$ if and only if $w \in \widetilde{W}^{ \pm}(v)$ respectively. We let $p_{ \pm}: \widetilde{\Sigma}_{k} \rightarrow \mathcal{L}^{ \pm}$denote the quotient maps, and give $\mathcal{L}^{ \pm}$the quotient topology induced by $p_{ \pm}$. For each $x \in \widetilde{M}$ the restriction $p_{+} \mid \widetilde{\Sigma}_{k} \cap T_{x} \widetilde{M}$ and $p_{-} \mid \widetilde{\Sigma}_{k} \cap T_{x} \widetilde{M}$ are homeomorphisms onto $\mathcal{L}^{+}$and $\mathcal{L}^{-}$respectively; thus $\mathcal{L}^{+}$and $\mathcal{L}^{-}$both admit the structure of topological manifolds, and are homeomorphic to $S^{n-1}$.

The key fact that we will need is that there exists a homeomorphism $F: \mathcal{L}^{+} \rightarrow$ $\mathcal{L}^{-}$with the following property: given any leaves $\widetilde{W}^{+} \in \mathcal{L}^{+}$and $\widetilde{W}^{-} \in \mathcal{L}^{-}$, the intersection $\widetilde{W}^{+} \cap \widetilde{W}^{-}$contains a unique flow line of $\widetilde{\phi}_{t}^{1}$ unless $\widetilde{W}^{-}=F\left(\widetilde{W}^{+}\right)$, in which case $\widetilde{W}^{+} \cap \widetilde{W}^{-}=\emptyset$. We will call $F$ a flip map.

To prove this we take advantage of the fact that $\widetilde{M}$ admits a metric $g_{0}$ of negative curvature again. As before let $S^{g_{0}} \widetilde{M}$ denote the unit sphere bundle of $\left(\widetilde{M}, g_{0}\right)$ and $\psi_{t}: S^{g_{0}} \widetilde{M} \rightarrow S^{g_{0}} \widetilde{M}$ the geodesic flow of $\left(\widetilde{M}, g_{0}\right)$. Let $\mathcal{L}_{g_{0}}^{+}$and $\mathcal{L}_{g_{0}}^{-}$denote the space of stable and unstable leaves determined by $\psi_{t}$. Defining similar equivalence relations $\sim_{+}^{g_{0}}$ and $\sim_{-}^{g_{0}}$ on $S^{g_{0}} \widetilde{M}$, we can realise $\mathcal{L}_{g_{0}}^{+}$and $\mathcal{L}_{g_{0}}^{-}$as quotients of $S^{g_{0}} \widetilde{M}$; let $p_{+}^{g_{0}}: S^{g_{0}} \widetilde{M} \rightarrow \mathcal{L}_{g_{0}}^{+}$and $p_{-}^{g_{0}}: S^{g_{0}} \widetilde{M} \rightarrow \mathcal{L}_{g_{0}}^{-}$denote the projections. Theorem 4.2 ensures we have a homeomorphism $G=G_{\tau}: \widetilde{\Sigma}_{k} \rightarrow S^{g_{0}} \widetilde{M}$ that conjugates the orbits of $\widetilde{\phi}_{t}^{1}$ and $\psi_{t}$, and this conjugacy then induces maps $G_{+}: \mathcal{L}^{+} \rightarrow \mathcal{L}_{g_{0}}^{+}$and $G_{-}: \mathcal{L}^{-} \rightarrow \mathcal{L}_{g_{0}}^{-}$. It is well known that the map $S^{g_{0}} \widetilde{M} \rightarrow S^{g_{0}} \widetilde{M}$ sending $v \mapsto-v$ induces a flip map $F_{0}: \mathcal{L}_{g_{0}}^{+} \rightarrow \mathcal{L}_{g_{0}}^{-}$for the flow $\psi_{t}$, and thus if $F:=G_{-}^{-1} \circ F_{0} \circ G_{+}$ then $F: \mathcal{L}^{+} \rightarrow \mathcal{L}^{-}$is a flip map for $\widetilde{\phi}_{t}^{1}$.

Among other things, the existence of a flip map gives us another way to view the holonomy transport. Given $v \in \widetilde{\Sigma}_{k}$, the restriction $p_{+} \mid \widetilde{W}^{u}(v)$ is a homeomorphism onto the set $\mathcal{L}^{+} \backslash F\left(\widetilde{W}^{+}(v)\right)$. Thus there is a unique map $p_{+}^{v}: \widetilde{\Sigma}_{k} \backslash F\left(\widetilde{W}^{+}(v)\right) \rightarrow$ $\widetilde{W}^{u}(v)$ such that $p_{+} \circ p_{+}^{v}=p_{+}$. Given $w \in \widetilde{W}^{+}(v)$, if

$$
V:=\widetilde{W}^{u}(w) \backslash F\left(\widetilde{W}^{+}(w)\right)
$$

then it is easy to see that $p_{+}^{v} \mid V$ is precisely the restriction to $V$ of the holonomy map $\widetilde{\mathcal{H}}_{w, v}^{\gamma}: \widetilde{W}^{u}(w) \rightarrow \widetilde{W}^{u}(v)$. We will use this fact in the proof below.

Proof. (of Theorem 5.6) 
Fix $v \in \Sigma_{k}$, and suppose $X$ and $Y$ are parallel sections of $E^{u}$ over $W^{u}(v)$. Then $[X, Y]=0$ as $\nabla$ is torsion free when restricted to $W^{u}(v)$. Since $\mathbb{J}$ is parallel by the previous lemma, so are $\mathbb{J} X$ and $\mathbb{I} Y$; hence $[\mathbb{J} X, \mathbb{J} Y]=[\mathbb{J} X, Y]=[X, \mathbb{J} Y=0$; in other words the Nijenhuis tensor $N_{\mathbb{J}}$ of $\mathbb{J}$ vanishes, and consequently $\mathbb{J}$ is integrable (see for instance, [28, Chapter IX, Theorem 2.4]). Thus we obtain a complex structure on $W^{u}(v)$. Using the almost complex structure $\mathbb{J}^{\prime}$ defined in the previous lemma (which we shall now also refer to simply as $\mathbb{J}$ ) and repeating the same argument gives us complex structures on the stable manifolds $W^{s}(v)$.

Let us now pass to the universal cover. We have shown that each of the strong stable and unstable leaves $\widetilde{W}^{s}$ and $\widetilde{W} u$ admit $\pi_{1}(M)$-equivariant complex structures. We will use this to define the structure of a complex manifold on the spaces $\mathcal{L}^{+}$and $\mathcal{L}^{-}$of leaves. We have shown above that given $v \in \widetilde{\Sigma}_{k}$, the map

$$
p_{+} \mid \widetilde{W}^{u}(v): \widetilde{W}^{u}(v) \rightarrow U(v):=\mathcal{L}^{+} \backslash F\left(\widetilde{W}^{+}(v)\right)
$$

is a homeomorphism. Let $\varphi_{v}: U(v) \rightarrow \widetilde{W}^{u}(v) \cong \mathbb{C}^{m}$ (here $n=2 m+1$ ) denote the inverse map. We want to define the structure of a complex manifold on $\mathcal{L}^{+}$ by taking $\left\{\left(\varphi_{v}, U(v): v \in \widetilde{\Sigma}_{k}\right\}\right.$ to be an atlas. The fact that the overlap maps $\varphi_{v} \circ \varphi_{w}^{-1}$ are holomorphic where defined follows immediately from the fact that if $v \in \widetilde{\Sigma}_{k}, w \in \widetilde{W}^{+}(v)$, and $V \subseteq \widetilde{W}^{u}(v)$ is as defined above in (5.3) then

$$
d_{w}\left(p_{+}^{v} \mid V\right)=\widetilde{H}_{w, v} \mid V
$$

and thus $p_{+}^{v} \mid V$ is holomorphic, since the lifted complex structure $\widetilde{\mathbb{J}}$ is invariant under the holonomy transport maps.

Similarly $\mathcal{L}^{-}$admits the structure of a complex manifold. Note that by construction the complex structure on $\mathcal{L}^{+}$and $\mathcal{L}^{-}$is $\pi_{1}(M)$-equivariant. Next, we claim that the orbit space $\widetilde{\Sigma}_{k} / \widetilde{\phi}_{t}^{1}$ is homeomorphic to $\mathcal{L}^{+} \times \mathcal{L}^{-} \backslash K$ where $K$ is a closed set. Set

$$
\Delta:=\left\{\left(p_{+}^{g_{0}}(v), p_{-}^{g_{0}}(v)\right) \in \mathcal{L}_{g_{0}}^{+} \times \mathcal{L}_{g_{0}}^{-}: v \in S^{g_{0}} \widetilde{M}\right\} .
$$

The following is well known: given a flow line $C_{v}(t)=\psi_{t} v$, the map

$$
C_{v} \mapsto\left(p_{+}^{g_{0}}(v), F_{0}\left(p_{-}^{g_{0}}(v)\right)\right) \in \mathcal{L}_{g_{0}}^{+} \times \mathcal{L}_{g_{0}}^{-}
$$

defines a homeomorphism between the orbit space $S^{g_{0}} \widetilde{M} / \psi_{t}$ of $\psi_{t}$ and $\mathcal{L}_{g_{0}}^{+} \times \mathcal{L}_{g_{0}}^{-} \backslash \Delta$. It follows that if

$$
K:=\left\{\left(p_{+}(v), p_{-}(v)\right) \in \mathcal{L}^{+} \times \mathcal{L}^{-}: v \in \widetilde{\Sigma}_{k}\right\},
$$

then given a flow line $\Gamma_{v}(t)=\widetilde{\phi}_{t}^{1} v$, the map

$$
\Gamma_{v} \mapsto\left(p_{+}(v), F\left(p_{-}(v)\right)\right) \in \mathcal{L}^{+} \times \mathcal{L}^{-}
$$

defines a homeomorphism from $\widetilde{\Sigma}_{k} / \widetilde{\phi}_{t}^{1}$ to $\mathcal{L}^{+} \times \mathcal{L}^{-} \backslash K$.

This essentially completes the proof. Indeed, we have shown that $\mathcal{L}^{+}$and $\mathcal{L}^{-}$ carry $\pi_{1}(M)$-equivariant complex structures, that the orbit space $\widetilde{\Sigma}_{k} / \widetilde{\phi}_{t}^{1}$ is $C^{1}$ equivalent to the complex manifold $\mathcal{L}^{+} \times \mathcal{L}^{-} \backslash K$ provided with the product complex structure, and that this correspondence is equivariant under the action of $\pi_{1}(M)$. Thus $\Sigma_{k}$ carries a real analytic structure for which the bundles $E^{+}$and $E^{-}$are real analytic, which by construction is $C^{1}$ diffeomorphic to the underlying smooth structure on $\Sigma_{k}$.

Write $\rho_{0}=\sigma+i t$, so that $\mathbb{J}=\sigma^{-1}\left(L^{u}-t \mathrm{Id}\right)$. Thus $L^{u}=\sigma J+t \mathrm{Id}$, and thus if $K:=\left|\rho_{0}\right|^{-2} \cdot(-\sigma \mathbb{J}+t \mathrm{Id})$ then $L^{u} \circ K=K \circ L^{u}=\operatorname{Id}$. Then since $d \lambda(\cdot, \cdot)=\omega_{1}\left(L^{s}(\cdot), \cdot\right)$ and $\omega_{1}^{n-1} \neq 0$, it follows that $(d \lambda)^{n-1} \neq 0$ and thus $\lambda \wedge(d \lambda)^{n-1}$ is a volume form.

Now we quote the following theorem of Benoist, Foulon and Labourie ([3], Theorem 1). 
Theorem 5.7. Let $N$ be a closed manifold of odd dimension $2 n-1$ with $n \geq 3$ an odd integer. Let $\phi_{t}: N \rightarrow N$ be an Anosov flow with infinitesimal generator $F$. Suppose that the strong bundles $E^{s}$ and $E^{u}$ are of class $C^{\infty}$. Define a 1-form $\lambda$ by $\operatorname{ker} \lambda=E^{s} \oplus E^{u}$ and $\lambda(F)=1$ and suppose $\lambda \wedge(d \lambda)^{n-1}$ is a volume form. Then there exists a unique cohomology class $\eta \in H^{1}(N, \mathbb{R})$ and a closed 1 -form $\beta$ representing $\eta$ such that $1+\beta(F)>0$ and such that if $Y:=(1+\beta(F))^{-1} F$ then the flow of $Y$ is $C^{\infty}$ conjugate to the geodesic flow of a Riemannian manifold of constant negative curvature.

Thus we conclude that there exists a Riemannian manifold $\left(N, h_{0}\right)$ of constant negative curvature, a $C^{1}$-diffeomorphism $G: S N \rightarrow \Sigma_{k}$ and a constant $c>0$ such that

$$
\rho_{t} \circ G=G \circ \tau_{c t},
$$

where $\rho_{t}$ is the flow of the vector field $Y=\left(1+\beta\left(F_{1}\right)\right)^{-1} F_{1}$, where $\beta \in \Omega^{1}\left(\Sigma_{k}\right)$ is the closed 1-form given by the theorem above, and $\tau_{t}: S N \rightarrow S N$ is the geodesic flow of $N$. In fact, by the main result in [18, $G$ is of class $C^{2}$.

Remark 5.8. Actually for the case $n=3$ we could bypass the above and conclude immediately that $\phi_{t}^{1}$ is $C^{\infty}$-orbit equivalent to the geodesic flow of a closed threedimensional hyperbolic manifold using recent work of Fang 12. Namely, for $n=3$ (so $\operatorname{dim} E^{u}=2$ ), an almost complex structure is the 'same' as having a conformal structure, and thus the fact that $E^{u}$ admits a $\phi_{t}^{1}$-invariant almost complex structure $\mathbb{J}$ implies that $\phi_{t}^{1}$ is quasiconformal (see [12]). Then [12, Theorem 3] tells us that up to finite covers, $\phi_{t}^{1}$ is $C^{\infty}$-orbit equivalent to the suspension of a symplectic hyperbolic automorphism or to the geodesic flow of a closed three-dimensional hyperbolic manifold. The former is impossible by Theorem 4.2. This method does not appear to work for $n=7$ however.

Let $\Omega_{\text {inv }}^{2}\left(\Sigma_{k}\right)$ denote the set of 2 -forms on $\Sigma_{k}$ that are invariant under $\phi_{t}^{1}$. It is easy to see that 2 -forms invariant under $\phi_{t}^{1}$ are precisely the same as the 2 -forms invariant under $\rho_{t}$.

Similarly let $\Omega_{\text {inv }}^{2}(S N)$ denote the set of 2-forms on $S N$ that are invariant under $\tau_{t}$. Note that $\omega_{1}$ and $d \lambda$ both lie in $\Omega_{\text {inv }}^{2}\left(\Sigma_{k}\right)$, and hence by (5.4) the 2-forms $G^{*} \omega_{1}$ and $G^{*} d \lambda$ on $S N$ both lie in $\Omega_{\text {inv }}^{2}(S N)$.

The following result is due to Kanai ([24, Claim 3.3]).

Theorem 5.9. If $n \geq 3$ then $\Omega_{\text {inv }}^{2}(S N)$ is 1-dimensional (where $\operatorname{dim} N=2 n-1$ ), spanned by the canonical symplectic form $\omega_{N}$ on $T N$. If $n=2$ then $\Omega_{\mathrm{inv}}^{2}(S N)$ is 2-dimensional, spanned by $\omega_{N}$ and another 2 -form $\psi_{N}$. This form can be uniquely characterized as follows. Let $\Pi: S \mathbb{H}^{3} \rightarrow S N$ denote the universal covering of $S N$, where $\mathbb{H}^{3}$ denotes hyperbolic 3 -space. Then there exists a 2-form $\psi \in \Omega^{2}\left(S \mathbb{H}^{3}\right)$ which we will define below, and $\psi_{N}$ is then the unique 2-form on $S N$ such that $p^{*} \psi_{N}=\psi$.

The theorem immediately implies the result for the case $n=7$. Indeed, we deduce that $G^{*} \omega_{1}=c_{1} \omega_{N}$ and $G^{*} d \lambda=c_{2} \omega_{N}$ for two constants $c_{1}, c_{2} \in \mathbb{R}$ with $c_{1}, c_{2} \neq 0$, and from this it is clear that $d \lambda=\rho \omega_{1}$ for some constant non-zero $\rho \in \mathbb{R}$. The case $n=3$ still requires a little work though.

Now we define the 2-form $\psi$ on $S \mathbb{H}^{3}$ mentioned in the statement of Theorem 5.9 , The Jacobi equation for $\mathbb{H}^{3}$ is given by

$$
\ddot{J}-J=0,
$$


(where the dot denotes the covariant derivative along $\gamma$ ) and thus it is easy to see that given a geodesic $\gamma$, the normal Jacobi fields along $\gamma$ are linear combinations of the fields

$$
J(t)=e^{ \pm t} U(t)
$$

where $U(t)$ is any parallel normal vector field along $\gamma$. Let $\phi_{t}^{\mathbb{H}}$ denote the geodesic flow on $\mathbb{H}^{3}$. Then $\phi_{t}^{\mathbb{H}}$ is Anosov (see Example 2.3). Given $v \in S \mathbb{H}^{3}$, let $\gamma_{v}$ denote the unique geodesic adapted to $v$. Then, given $\xi \in T_{v} S \mathbb{H}^{3}$, let $J_{\xi}$ denote the unique Jacobi field along $\gamma_{v}$ with $J_{\xi}(0)=d_{v} \pi_{\mathbb{H}}(\xi)$ and $\dot{J}_{\xi}(0)=K_{\mathbb{H}, v}(\xi)$ (here $\pi_{\mathbb{H}}: S \mathbb{H}^{3} \rightarrow \mathbb{H}^{3}$ and $K_{\mathbb{H}}: T T \mathbb{H}^{3} \rightarrow T \mathbb{H}^{3}$ denote the footpoint and connection maps of $\mathbb{H}^{3}$ respectively.

Using the fact that $d \phi_{t}^{\mathbb{H}}(\xi)=\left(J_{\xi}(t), \dot{J}_{\xi}(t)\right)$ (see for instance [29, Section 1.5]), it easily follows that the Anosov splitting $S \mathbb{H}^{3}=\mathbb{R} F_{\mathbb{H}} \oplus E_{\mathbb{H}}^{s} \oplus E_{\mathbb{H}}^{u}$ is given by

$$
E_{\mathbb{H}}^{s}(v)=\left\{\xi \in T_{v} S \mathbb{H}^{3}: \xi_{H}=-\xi_{V}\right\},
$$

and similarly

$$
\begin{gathered}
E_{\mathbb{H}}^{u}(v)=\left\{\xi \in T_{v} S \mathbb{H}^{3}: \xi_{H}=\xi_{V}\right\}, \\
\mathbb{R} F_{\mathbb{H}}(v)=\left\{\xi \in T_{v} S \mathbb{H}^{3}: \xi_{V}=0, \xi_{H}=a v, a \in \mathbb{R}\right\} .
\end{gathered}
$$

Using this decomposition we can define an almost complex structure on the subbundle $E_{\mathbb{H}}=E_{\mathbb{H}}^{s} \oplus E_{\mathbb{H}}^{u}$. Indeed, fix $v \in S \mathbb{H}^{3}$ and note that the isomorphism

$$
T_{v} T \mathbb{H}^{3} \cong T_{x} \mathbb{H}^{3} \oplus T_{x} \mathbb{H}^{3}
$$

described at the beginning of Section 4 restricts to define an isomorphism

$$
E_{\mathbb{H}}(v) \cong v^{\perp} \oplus v^{\perp},
$$

where

$$
v^{\perp}:=\left\{w \in T_{x} \mathbb{H}^{3}:\langle w, v\rangle=0\right\} .
$$

Now $v^{\perp}$ is 2-dimensional, and let $\left\{e_{1}(v), e_{2}(v)\right\} \subset v^{\perp}$ be an orthonormal basis such that $\left\{e_{1}(v), e_{2}(v), v\right\}$ is a positively oriented basis of $T_{x} \mathbb{H}^{3}$. This allows us to define a map $i_{v}: v^{\perp} \rightarrow v^{\perp}$ by $i_{v} e_{1}(v)=e_{2}(v)$ and $i_{v} e_{2}(v)=-e_{1}(v)$.

Now define $\mathbb{J}_{v}: E_{\mathbb{H}}^{s}(v) \rightarrow E_{\mathbb{H}}^{s}(v)$ by

$$
\mathbb{J}_{v}\left(\xi_{H},-\xi_{H}\right)=\left(i_{v} \xi_{H},-i_{v} \xi_{H}\right),
$$

and define $\mathbb{J}_{v}: E_{\mathbb{H}}^{u}(v) \rightarrow E_{\mathbb{H}}^{u}(v)$ by

$$
\mathbb{J}_{v}\left(\xi_{H}, \xi_{H}\right)=\left(-i_{v} \xi_{H},-i_{v} \xi_{H}\right) .
$$

This defines an almost complex structure on $E_{\mathbb{H}}$. For convenience, extend $\mathbb{J}$ to all of $T S \mathbb{H}^{3}$ by letting $\mathbb{J} \mid \mathbb{R} F_{\mathbb{H}} \equiv 0$.

Finally, we define $\psi \in \Omega^{2}\left(S \mathbb{H}^{3}\right)$ by

$$
\psi_{v}(\xi, \eta)=\left.\omega_{\mathbb{H}}\right|_{v}\left(\mathbb{J}_{v} \xi, \eta\right) \quad \text { for } \quad \xi, \eta \in T_{v} S \mathbb{H}^{3} .
$$

This form $\psi$ is the 2-form referred in the statement of Theorem 5.9. Note that $\psi$ is closed because $d \psi$ is an invariant 3-form which must vanish by the proof of Lemma 3.1 .

The sphere bundle $S \mathbb{H}^{3}$ is trivial: $S \mathbb{H}^{3}=\mathbb{H}^{3} \times S^{2}$. Given $x \in \mathbb{H}^{3}$, let $S_{x}$ be the 2 -sphere of unit vectors at $x$. Let us make the following observation which shows that $[\psi] \in H^{2}\left(S \mathbb{H}^{3}, \mathbb{R}\right)=\mathbb{R}$ is not zero.

Lemma 5.10. For all $x \in \mathbb{H}^{3}$ we have

$$
\int_{S_{x}} \psi \neq 0
$$


Proof. Fix $x \in \mathbb{H}^{3}$ and $v \in S_{x} \mathbb{H}^{3}$. Let us take two vectors $\xi, \eta \in V(v)$, with say,

$$
\xi=\left(0, \xi_{V}\right) \in T_{x} \mathbb{H}^{3} \oplus T_{x} \mathbb{H}^{3}, \quad \eta=\left(0, \eta_{V}\right) \in T_{x} \mathbb{H}^{3} \oplus T_{x} \mathbb{H}^{3} .
$$

It suffices to observe that $\psi_{v}(\xi, \eta) \neq 0$ if $\xi$ and $\eta$ are not colinear. We need to express $\xi$ as a sum of elements of $E_{\mathbb{H}}^{s}(v)$ and $E_{\mathbb{H}}^{u}(v)$ : this is easily done by noting

$$
\xi=\underbrace{\frac{1}{2}\left(-\xi_{V}, \xi_{V}\right)}_{\in E_{\mathbb{H}}^{s}(v)}+\underbrace{\frac{1}{2}\left(\xi_{V}, \xi_{V}\right)}_{\in E_{\mathbb{H}}^{u}(v)} .
$$

Then we have

$$
\mathbb{J}_{v}(\xi)=\frac{1}{2}\left(-i_{v} \xi_{V},+i_{v} \xi_{V}\right)+\frac{1}{2}\left(-i_{v} \xi_{V},-i_{v} \xi_{V}\right)=-\left(i_{v} \xi_{V}, 0\right)
$$

Thus:

$$
\begin{aligned}
\psi_{v}(\xi, \eta) & =\left.\omega_{\mathbb{H}}\right|_{v}\left(\mathbb{J}_{v} \xi, \eta\right) \\
& =-\left\langle i_{v} \xi_{V}, \eta_{V}\right\rangle,
\end{aligned}
$$

and this is non-zero if $\xi$ and $\eta$ are not colinear.

From this it is now easy to complete the proof in the case $n=3$. Since both $\omega_{0}$ and $d \lambda$ are exact (recall $\omega_{0}=-d \alpha$; see (1.1)) it follows that both $\Pi^{*} G^{*} \omega_{1}$ and $\Pi^{*} G^{*} d \lambda$ vanish in $H^{2}\left(S \mathbb{H}^{3}, \mathbb{R}\right)$ (since $\omega_{1}$ is equal to $\omega_{0}$ plus the pullback of a form on the base), and thus they must both be multiples of $\omega_{\mathbb{H}}$; that is,

$$
\Pi^{*} G^{*} \omega_{1}=c_{1} \omega_{\mathbb{H}}, \quad \Pi^{*} G^{*} d \lambda=c_{2} \omega_{\mathbb{H}}: c_{1}, c_{2} \in \mathbb{R} \backslash\{0\} .
$$

Hence again $d \lambda=\rho \omega_{1}$ for some non-zero $\rho \in \mathbb{R}$, which completes the proof for the case $n=3$, and thus finally completes the proof of Theorem A.

Proof of the Corollary B. Now we prove Corollary B from the introduction.

Proposition 5.11. Suppose $g$ is negatively curved and strictly 1/4-pinched. For sufficiently large $k$, the Hamiltonian structure $\left(\Sigma_{k}, \omega_{1}\right)$ is Anosov and satisfies the 1/2-pinching condition.

Proof. First of all we will show that $\left(\Sigma_{k}, \omega_{1}\right)$ is an Anosov Hamiltonian structure for $k$ sufficiently large. It is well known that $\phi_{t}^{0}: S M \rightarrow S M$ is Anosov. By structural stability of Anosov flows, there exists $\delta_{0}>0$ such that $\phi_{t}^{\varepsilon}: S M \rightarrow S M$ is Anosov for all $\varepsilon \in\left(-\delta_{0}, \delta_{0}\right)$ (see for instance [25, Corollary 18.2.2]).

Consider now the map

$$
h_{\varepsilon}: T M \rightarrow T M, v \mapsto \varepsilon v .
$$

Then the observation is that

$$
h_{\varepsilon}^{*} \omega_{\varepsilon}=\varepsilon \omega_{1}, \quad h_{\varepsilon}^{*} H=\varepsilon^{2} H .
$$

Now $\phi_{t}^{\varepsilon}: S M \rightarrow S M$ is Anosov if and only if the flow (temporarily called) $\psi_{t}$ of $h_{\varepsilon}^{*} H$ with respect to $h_{\varepsilon}^{*} \omega_{\varepsilon}$ is Anosov on $\left(h_{\varepsilon}^{*} H\right)^{-1}(1 / 2)$. But $\psi_{t}$ is the Hamiltonian flow of $(x, v) \mapsto \frac{\varepsilon^{2}}{2}|v|^{2}$ with respect to the symplectic form $\varepsilon \omega_{1}$. But it is easy to see that $\psi_{t}=\phi_{\varepsilon t}^{1}$; hence we have shown that

$$
\phi_{t}^{\varepsilon}: S M \rightarrow S M \text { is Anosov } \Leftrightarrow \phi_{t}^{1}: \Sigma_{1 / 2 \varepsilon^{2}} \rightarrow \Sigma_{1 / 2 \varepsilon^{2}} \text { is Anosov. }
$$

Thus in particular for

$$
k>\frac{1}{2} \delta_{0}^{-2},
$$

$\left(\Sigma_{k}, \omega_{1}\right)$ is an Anosov Hamiltonian structure.

In order to prove that $\left(\Sigma_{k}, \omega_{1}\right)$ is $1 / 2$-pinched for $k$ large we note that an equivalent claim is that $\left(\Sigma_{1 / 2}, \omega_{\varepsilon \sigma}\right)$ is $1 / 2$-pinched for small $\varepsilon$. An inspection of the proof of (2.1) and (2.2) in [26, Theorem 3.2.17] (see Example 2.3) shows that if we do 
the same analysis for the magnetic Jacobi (or Riccati) equation we obtain numbers $k_{1}(\varepsilon)$ and $k_{0}(\varepsilon)$ for which (2.1) and (2.2) hold. These numbers will be as close as we wish to $k_{1}(0)=2$ and $k_{0}(0)=\sqrt{-\max K}>1$ if $\varepsilon$ is small enough and the 1/2-pinching condition follows (see Example 2.3 again).

From this it is easy to prove Corollary B. Suppose $k$ is chosen large enough such that $\left(\Sigma_{k}, \omega_{1}\right)$ is an Anosov Hamiltonian structure satisfying the $1 / 2$-pinching condition, and suppose for contradiction that $\left(\Sigma_{k}, \omega_{1}\right)$ is stable, and let $\lambda$ be a stabilizing 1-form. By Theorem A, $\left(\Sigma_{k}, \omega_{1}\right)$ is contact, that is, $d \lambda=\rho \omega_{1}$ for some non-zero $\rho \in \mathbb{R}$. In particular, $\omega_{1}$ is exact which in turn implies that $\sigma$ is exact.

\section{REFERENCES}

1. D. Anosov, Geodesic flows on closed Riemannian manifolds with negative curvature, Trudy Math. Inst. Steklov 90 (1967).

2. V. I. Arnold and A. B. Givental, Symplectic Geometry, Dynamical Systems, Encyclopedia of Mathematical Sciences, vol. IV, Springer-Verlag, 1990.

3. Y. Benoist, P. Foulon, and F. Labourie, Flots d'Anosov à distributions stable et instable différentiables, Jour. Amer. Math. Soc. 5 (1992), no. 1, 33-74.

4. F. Bourgeois, Y. Eliashberg, H. Hofer, K. Wysocki, and E. Zehnder, Compactness results in symplectic field theory, Geom. Topol. 7 (2003), 799-888.

5. K. Burns and G. P. Paternain, Anosov magnetic flows, critical values and topological entropy, Nonlinearity 15 (2002), 281-314.

6. C. Camacho and A. Lins Neto, Geometric Theory of Foliations, Birkhäuser Verlag, 1985.

7. K. Cieliebak, U. Frauenfelder, and G. P. Paternain, Symplectic topology of Mañé's critical value, To appear in Geometry and Topology, arXiv:0903.0700 (2009).

8. K. Cieliebak and K. Mohnke, Compactness for punctured holomorphic curves. Conference on Symplectic Topology, J. Symp. Geom. 3 (2005), no. 4, 589-654.

9. G. Contreras, R. Iturriaga, G. P. Paternain, and M. Paternain, Lagrangian graphs, minimizing measures and Mañé's critical values, GAFA 8 (1998), 788-809.

10. R. de la Llave, J. Marco, and R. Moryion, Canonical Perturbation Theory of Anosov Systems and Regularity Results for the Livsic Cohomology Equation, Ann. Math. 123 (1986), 537-611.

11. Y. Eliashberg, S.S. Kim, and L. Polterovich, Geometry of contact transformations and domains: orderability versus squeezing, Geom. Topol. 10 (2006), 1635-1747.

12. Y. Fang, On the rigidity of quasiconformal Anosov flows, Ergod. Th. \& Dynam. Sys. 27 (2007), 1773-1802.

13. R. Feres, Geodesic flows on manifolds of negative curvature with smooth horospheric foliations, Ergod. Th. \& Dynam. Sys. 11 (1991), 653-686.

14. R. Feres and A. Katok, Anosov flows with smooth foliations and rigidity of geodesic flows on three-dimensional manifolds of negative curvature, Ergod. Th. \& Dynam. Sys. 10 (1990), 657-670.

15. P. Foulon, Feuilletages des sphères et dynamiques Nord-Sud, C. R. Acad. Sci. Paris 318 (1994), 1041-1042.

16. É. Ghys, Flots d'Anosov sur les 3-variétés fibrées en cercles, Ergod. Th. \& Dynam. Sys. 4 (1984), 67-80.

17. M. Gromov, Three remarks on geodesic dynamics and the fundamental group, Enseign Math. (2) 46 (2000), no. 3-4, 391-402.

18. U. Hamenstädt, Regularity of time preserving conjugacies for contact Anosov flows with $C^{1}$ Anosov splitting, Ergod. Th. \& Dynam. Sys. 13 (1993), 65-72.

19. Invariant two-forms for geodesic flows, Math. Ann. 301 (1995), 677-698.

20. B. Hasselblatt, Regularity of the Anosov splitting and of horospheric foliations, Ergod. Th. \& Dynam. Sys. 14 (1994), 645-666.

21. M. Hirsch, C. Pugh, and M. Shub, Invariant manifolds, Lec. Notes in Math., vol. 583, SpringerVerlag, 1977.

22. H. Hofer and E. Zehnder, Symplectic invariants and Hamiltonian dynamics, Birkhäuser Verlag, 1994.

23. M. Kanai, Geodesic flows of negatively curved manifolds with smooth stable and unstable foliations, Ergod. Th. \& Dynam. Sys. 8 (1988), 215-239.

24. _ Differential-geometric studies on dynamics of geodesic and frame flows, Japan. J. Math. 19 (1993), no. 1, 1-29. 
25. A. Katok and B. Hasselblatt, Introduction to the Modern Theory of Dynamical Systems, Encyclopedia of Mathematics and its Applications, vol. 54, Cambridge University Press, 1995.

26. W. Klingenberg, Riemannian Geometry, 2 ed., de Gruyter Studies in Math., no. 1, Walter de Gruyter \& Co, 1995.

27. G. Knieper, Hyperbolic Dynamics and Riemannian geometry, Handbook of Dynamical Systems (B. Hasselblatt and A. Katok, eds.), vol. IA, Elsevier, 2002.

28. S. Kobayashi and K. Nomizu, Foundations of Differential Geometry, vol. 2, Wiley, 1963.

29. G. P. Paternain, Geodesic Flows, vol. 180, Birkhäuser Verlag, 1999.

30. G. P. Paternain and M. Paternain, On Anosov energy levels of convex Hamiltonian systems, Math. Zeitschrift 217 (1994), 367-376.

31. N. Peyerimhoff and K. F. Siburg, The dynamics of magnetic flows for energies above Mañé's critical value, Isr. J. Math. 135 (2003), 269-298.

32. N. Steenrod, The Topology of Fibre Bundles, Princeton University Press, 1951.

Department of Pure Mathematics and Mathematical Statistics, University of CamBridge, Cambridge CB3 0WB, England

E-mail address: w.merry@dpmms.cam.ac.uk, g.p.paternain@dpmms.cam.ac.uk 